\title{
Asymptotic Behavior of Stochastic Fractional Power Dissipative Equations on $\mathbb{R}^{n}$
}

\author{
Hong Lu, Peter W. Bates, ${ }^{\dagger}$ Jie Xin $\ddagger$ and Mingji Zhang ${ }^{\S}$
}

\begin{abstract}
We study a reaction-fractional diffusion equation with additive noise on the entire space $\mathbb{R}^{n}$ with particular interest in the asymptotic behavior of solutions. We first transform the equation into a random equation whose solutions generate a random dynamical system. A priori estimates for solutions are derived when the nonlinearity satisfies certain growth conditions. Using estimates for far-field values of solutions and a cut-off technique, asymptotic compactness is proved. Thus, the existence of a random attractor in $L^{2}\left(\mathbb{R}^{n}\right)$ is established.
\end{abstract}

Keywords stochastic equation, fractional Laplacian, asymptotic compactness, random attractor, pullback attractor

MSC: 37L55; 35B40; 35B41

\section{Introduction}

Fractional derivative operators, modeling non-standard diffusive processes, arise in a wide range of fields within physics, biology, chemistry, etc. Specific examples include kinetic theories of systems with chaotic dynamics $([29,37])$, pseudochaotic dynamics ([38]), dynamics in a complex or porous medium $([12,22,30])$, random walks with a memory and flights $([20,28,36])$, and obstacle problems $([6,26])$. Recently, some of the classical equations of mathematical physics

\footnotetext{
${ }^{*}$ College of Science, China University of Mining and Technology, Jiangsu 221116, China, E-mail: ljwenling@163.com

${ }^{\dagger}$ Department of Mathematics, Michigan State University, East Lansing, MI 48824, USA, E-mail: bates@math.msu.edu

${ }^{\ddagger}$ School of Mathematics and Statistics Science, Ludong University, Yantai, Shandong 100191, China, E-mail: fdxinjie@sina.com

${ }^{\S}$ Department of Mathematics, New Mexico Institute of Mining and Technology, Socorro, NM 87801, USA, E-mail: mzhang@nmt.edu.
} 
have been postulated with fractional derivatives to better describe complex phenomena (e.g., $[11,15,16,17,24,32])$.

Small perturbations (such as molecular collisions in gases and liquids and electric fluctuations in resistors [14]) may be neglected during the derivation of these ideal models. However, these stochastic perturbations should be included to obtain a more realistic model and to better understand the dynamical behavior of the phenomena being studied. In may cases one may represent the micro effects by random perturbations in the dynamics of the macro observable through multiplicative or additive noise in the governing equation. In these cases, one may reformulate the equation as a random dynamical system, as shown below.

A key step to study a stochastic partial differential equation is to examine the asymptotic behavior of the random dynamical systems generated by its solutions. Some fundamental advances in this direction may be found, for example, in papers by Crauel and Flandoli $([7,8])$ who developed the theory of random attractors in a manner which closely parallels the deterministic case ([31]). Also, Debussche ([10]) proved that the Hausdorff dimension of the random attractor could be estimated by using global Lyapunov exponents.

The well-posedness of solutions of fractional partial differential equations has been studied to some extent in special equations (e.g., $[16,17,19,24])$. The Cauchy problem for the semilinear fractional power dissipative equation

$$
\begin{cases}u_{t}+(-\triangle)^{\alpha} u=F(u), & (t, x) \in \mathbb{R}^{+} \times \mathbb{R}^{n} \\ u(x, 0)=u_{0}(x), & x \in \mathbb{R}^{n}\end{cases}
$$

is studied in [21] and [35], where the general equation in (1.1) includes, as special cases, several classical equations. For example, the dissipative quasi-geostrophic (QG) equation, a generalized Navier-Stokes equation, a generalized convection-diffusion equation, and the Ginzburg-Landau equation (see [21] for more details), are all included.

However, there are not many results for stochastic fractional partial differential equations. In this paper, we examine the asymptotic behavior of solutions of the following stochastic semilinear fractional power dissipative equation with additive noise:

$$
d u+\left((-\triangle)^{\alpha} u+\lambda u\right) d t=F(x, u) d t+\sum_{j=1}^{m} \phi_{j} d \omega_{j}(t), \quad x \in \mathbb{R}^{n}, \quad t>0
$$

with the initial condition

$$
u(x, 0)=u_{0}(x), \quad x \in \mathbb{R}^{n},
$$


where $\alpha \in(1 / 2,1)$ and $\lambda$ is a positive constant. The functions $\left\{\phi_{j}\right\}_{j=1}^{m} \subset H^{2}\left(\mathbb{R}^{n}\right) \cap W^{2, p}\left(\mathbb{R}^{n}\right)$ for some $p>1$ and $\left\{\omega_{j}\right\}_{j=1}^{m}$ are independent two-sided real-valued Wiener processes on a complete probability space. These result from the possibility that small irregularities, stochastic in time, should be taken into account. In the various lemmas that follow we assume that $F(x, s)$ is continuous and satisfies some of the following conditions:

$$
\begin{aligned}
F(x, s) s & \leq-\beta_{1}|s|^{p}+\gamma_{1}(x) \\
|F(x, s)| & \leq \beta_{2}|s|^{p-1}+\gamma_{2}(x), \\
\frac{\partial}{\partial s} F(x, s) & \leq \beta_{3}, \\
\left|\frac{\partial}{\partial x} F(x, s)\right| & \leq \gamma_{3}(x),
\end{aligned}
$$

where $p>1$, as above, and $\beta_{i}(i=1,2,3)$ are positive constants, $\gamma_{1}(x) \in L^{1}\left(\mathbb{R}^{n}\right) \cap L^{\infty}\left(\mathbb{R}^{n}\right)$, $\gamma_{2}(x) \in L^{2}\left(\mathbb{R}^{n}\right) \cap L^{q}\left(\mathbb{R}^{n}\right)$ with $\frac{1}{q}+\frac{1}{p}=1$, and $\gamma_{3}(x) \in L^{2}\left(\mathbb{R}^{n}\right)$.

The concept of pullback random attractor, an extension of global attractor in deterministic systems (see $[2,18,25,27,31])$, was introduced in [8, 13]. In the case of bounded domains, the existence of random attractors for stochastic partial differential equations has been investigated by many authors (see $[1,7,8,9,13]$ and the references therein). However, the problem is more challenging for unbounded domains. Recently, the existence of random attractors for systems on unbounded domains was studied in $[3,5,33,34]$, which provided guidance for this work.

It is well known that the asymptotic compactness and the existence of a bounded absorbing set are sufficient to guarantee the existence of a random attractor for a continuous random dynamic system. Here, to achieve these goals, we employ estimates on far-field values of solutions.

The paper is organized as follows. In section 2, some preliminaries, notation and random attractor theory for random dynamical systems are introduced. In section 3, we produce a continuous random dynamical system to represent the stochastic fractional power dissipative equation. In section 4, we derive uniform estimates for solutions, which include the uniform estimates on far-field values of solutions in the $L^{2}$ sense. The existence of a pullback random attractor is established in section 5 . 


\section{Preliminaries and Notations}

We first recall some basic concepts related to random attractors for stochastic dynamical systems (see $[4,8,9]$ for more details).

Let $\left(X,\|\cdot\|_{X}\right)$ be a separable Hilbert space with Borel $\sigma$-algebra $\mathcal{B}(X)$, and let $(\Omega, \mathcal{F}, \mathbb{P})$ be a probability space.

Definition 2.1. $\left(\Omega, \mathcal{F}, \mathbb{P},\left(\theta_{t}\right)_{t \in \mathbb{R}}\right)$ is called a measurable dynamical system, if $\theta: \mathbb{R} \times \Omega \rightarrow \Omega$ is $(\mathcal{B}(\mathbb{R}) \times \mathcal{F}, \mathcal{F})$-measurable, $\theta_{0}=\mathbb{I}, \theta_{t+s}=\theta_{t} \circ \theta_{s}$ for all $t, s \in \mathbb{R}$, and $\mathbb{P}\left(\theta_{t} A\right)=\mathbb{P}(A)$ for all $t \in \mathbb{R}$ and $A \in \mathcal{F}$.

Definition 2.2. A stochastic process $\phi(t, \omega)$ is called a continuous random dynamical system (RDS) over the measurable dynamical system $\left(\Omega, \mathcal{F}, \mathbb{P},\left(\theta_{t}\right)_{t \in \mathbb{R}}\right)$ if $\phi$ is $\left(\mathcal{B}\left(\mathbb{R}^{+}\right) \times \mathcal{F} \times \mathcal{B}(X), \mathcal{B}(X)\right)$-measurable, and for all $\omega \in \Omega$,

(i) the mapping $\phi: \mathbb{R}^{+} \times \Omega \times X \rightarrow X$ is continuous;

(ii) $\phi(0, \omega)=\mathbb{I}$ on $X$;

(iii) $\phi(t+s, \omega, \chi)=\phi\left(t, \theta_{s} \omega, \phi(s, \omega, \chi)\right)$ for all $t, s \geq 0$ and $\chi \in X$ (cocycle property).

Definition 2.3. A random bounded set $\{B(\omega)\}_{\omega \in \Omega} \subseteq X$ is called tempered with respect to $\left(\theta_{t}\right)_{t \in \mathbb{R}}$ if for P-a.e. $\omega \in \Omega$ and all $\epsilon>0$,

$$
\lim _{t \rightarrow \infty} e^{-\epsilon t} d\left(B\left(\theta_{-t} \omega\right)\right)=0
$$

where $d(B)=\sup _{\chi \in B}\|\chi\|_{X}$.

Let $\phi(t, w)$ be a continuous random dynamical system over $\left(\Omega, \mathcal{F}, \mathbb{P},\left(\theta_{t}\right)_{t \in \mathbb{R}}\right)$, and let $\mathcal{D}$ be the collection of all tempered random subsets of $X$.

Definition 2.4. $\mathcal{D}$ is called inclusion-closed if $D=\{D(\omega)\}_{\omega \in \Omega} \in \mathcal{D}$ and if $\tilde{D}=\{\tilde{D}(\omega)\}_{\omega \in \Omega}$ is a random subset of $X$ with $\tilde{D}(\omega) \subseteq D(\omega)$ for all $\omega \in \Omega$ then $\tilde{D} \in \mathcal{D}$.

Definition 2.5. Let $\mathcal{D}$ be a collection of random subsets of $X$ and $\{K(\omega)\}_{\omega \in \Omega} \in \mathcal{D}$. Then $\{K(\omega)\}_{\omega \in \Omega}$ is called an absorbing set of $\phi$ in $\mathcal{D}$ if for all $B \in \mathcal{D}$ and P-a.e. $\omega \in \Omega$, there exists $t_{B}(\omega)>0$ such that

$$
\phi\left(t, \theta_{-t} \omega, B\left(\theta_{-t} \omega\right)\right) \subseteq K(\omega), \quad t \geq t_{B}(\omega)
$$


Definition 2.6. Let $\mathcal{D}$ be a collection of random subsets of $X$. Then $\phi$ is said to be $\mathcal{D}$-pullback asymptotically compact in $\mathbf{X}$ if for P-a.e. $\omega \in \Omega,\left\{\phi\left(t_{n}, \theta_{-t_{n}} \omega, \chi_{n}\right)\right\}_{n=1}^{\infty}$ has a convergent subsequence in $X$ whenever $t_{n} \rightarrow \infty$, and $\chi_{n} \in B\left(\theta_{-t_{n}} \omega\right)$ with $\{B(\omega)\}_{\omega \in \Omega} \in \mathcal{D}$.

Definition 2.7. Let $\mathcal{D}$ be a collection of random subsets of $X$ and $\{\mathcal{A}(\omega)\}_{\omega \in \Omega} \in \mathcal{D}$. Then $\{\mathcal{A}(\omega)\}_{\omega \in \Omega}$ is called a $\mathcal{D}$-random attractor (or D-pullback attractor) for $\phi$ if the following conditions are satisfied, for P-a.e. $\omega \in \Omega$,

(i) $\mathcal{A}(\omega)$ is compact, and $\omega \rightarrow d(\chi, \mathcal{A}(\omega))$ is measurable for every $\chi \in X$;

(ii) $\{\mathcal{A}(\omega)\}_{\omega \in \Omega}$ is strictly invariant, i.e., $\phi(t, \omega, \mathcal{A}(\omega))=\mathcal{A}\left(\theta_{t} \omega\right), \forall t \geq 0$ and for a.e. $\omega \in \Omega$;

(iii) $\{\mathcal{A}(\omega)\}_{\omega \in \Omega}$ attracts all sets in $\mathcal{D}$, i.e., for all $B \in \mathcal{D}$ and a.e. $\omega \in \Omega$, we have

$$
\lim _{t \rightarrow \infty} d\left(\phi\left(t, \theta_{-t} \omega, B\left(\theta_{-t} \omega\right)\right), \mathcal{A}(\omega)\right)=0
$$

where $d$ is the Hausdorff semi-metric given by $d(Y, Z)=\sup _{y \in Y} \inf _{z \in Z}\|y-z\|_{X}$, for any $Y, Z \subseteq X$.

From [13], we have the following result.

Proposition 2.1. Let $\mathcal{D}$ be an inclusion-closed collection of random subsets of $X$ and $\phi$ a continuous RDS on $X$ over $\left(\Omega, \mathcal{F}, \mathbb{P},\left(\theta_{t}\right)_{t \in \mathbb{R}}\right)$. Suppose that $\{K(\omega)\}_{\omega \in \Omega} \in \mathcal{D}$ is a closed absorbing set for $\phi$ and $\phi$ is $\mathcal{D}$-pullback asymptotically compact in $X$. Then $\phi$ has a unique $\mathcal{D}$-random attractor $\{\mathcal{A}(\omega)\}_{\omega \in \Omega}$ given by

$$
\mathcal{A}(\omega)=\bigcap_{\kappa \geq 0} \overline{\bigcup_{t \geq \kappa} \phi\left(t, \theta_{-t} \omega, K\left(\theta_{-t} \omega\right)\right)} .
$$

For convenience, we recall some notation related to the fractional derivative and fractional Sobolev spaces. We first present the definition and the properties of $(-\triangle)^{\alpha}$ through the Fourier transform ([17]). We consider the Fourier transform

$$
\Phi(\xi)=\mathcal{F}(\phi)(\xi)=\hat{\phi}(\xi)=\int_{\mathbb{R}^{n}} \phi(x) e^{-\mathrm{i} x \cdot \xi} d x,
$$

so $(-\triangle)^{\frac{\beta}{2}}$ can be defined as

$$
\begin{aligned}
\mathcal{F}\left\{(-\triangle)^{\frac{\beta}{2}} \varphi\right\} & =|\xi|^{\beta} \Phi \\
(-\triangle)^{\frac{\beta}{2}} \varphi & =\mathcal{F}^{-1}\left\{|\xi|^{\beta} \Phi\right\}=\frac{1}{(2 \pi)^{n}} \int_{\mathbb{R}^{n}}|\xi|^{\beta} \Phi e^{\mathrm{i} \xi \cdot x} d \xi .
\end{aligned}
$$

Let $H^{\beta}\left(\mathbb{R}^{n}\right)$ denote the Sobolev space of order $\beta$ under the norm:

$$
\|\phi\|_{H^{\beta}}^{2}=\int_{\mathbb{R}^{n}}\left(1+|\xi|^{2 \beta}\right)|\Phi(\xi)|^{2} d \xi
$$

By virtue of the definition of $(-\triangle)^{\alpha}$, we have the following formula for integration by parts. 
Lemma 2.1. If $f, g \in H^{2 \alpha}\left(\mathbb{R}^{n}\right)$, then the following equation holds.

$$
\int_{\mathbb{R}^{n}}(-\triangle)^{\alpha} f \cdot g d x=\int_{\mathbb{R}^{n}}(-\triangle)^{\alpha_{1}} f \cdot(-\triangle)^{\alpha_{2}} g d x,
$$

where $\alpha_{1}$ and $\alpha_{2}$ are nonnegative constants and satisfy $\alpha_{1}+\alpha_{2}=\alpha$.

Proof. By the definition of $(-\triangle)^{\alpha}$ and Parseval formula, we have

$$
\begin{aligned}
\int_{\mathbb{R}^{n}}(-\triangle)^{\alpha} f \cdot g d x & =\int_{\mathbb{R}^{n}} \mathcal{F}^{-1}\left\{|k|^{2 \alpha} \hat{f}\right\} \cdot g d x=\int_{\mathbb{R}^{n}} \mathcal{F}^{-1}\left\{|k|^{2 \alpha} \hat{f}\right\} \cdot \mathcal{F}^{-1} \hat{g} d x \\
& =\frac{1}{(2 \pi)^{n}} \int_{\mathbb{R}^{n}}|k|^{2 \alpha} \hat{f} \cdot \hat{g} d k=\frac{1}{(2 \pi)^{n}} \int_{\mathbb{R}^{n}}|k|^{2 \alpha_{1}} \hat{f} \cdot|k|^{2 \alpha_{2}} \hat{g} d k \\
& =\int_{\mathbb{R}^{n}} \mathcal{F}^{-1}\left\{|k|^{2 \alpha_{1}} \hat{f}\right\} \cdot \mathcal{F}^{-1}\left\{|k|^{2 \alpha_{2}} \hat{g}\right\} d x=\int_{\mathbb{R}^{n}}(-\triangle)^{\alpha_{1}} f \cdot(-\triangle)^{\alpha_{2}} g d x .
\end{aligned}
$$

In addition, the Gagliardo-Nirenberg inequality ([23]) and Gronwall's Lemma, given below, are also frequently used throughout this paper.

Lemma 2.2. Let $u$ belong to $L^{q}\left(\mathbb{R}^{n}\right)$ and its derivatives of order $m, D^{m} u$, belong to $L^{r}\left(\mathbb{R}^{n}\right), 1 \leq q, r \leq \infty$. For the derivatives $D^{j} u, 0 \leq j<m$, the following inequality holds

$$
\left\|D^{j} u\right\|_{L^{p}} \leq c\left\|D^{m} u\right\|_{L^{r}}^{\theta}\|u\|_{L^{q}}^{1-\theta}
$$

where

$$
\frac{1}{p}=\frac{j}{n}+\theta\left(\frac{1}{r}-\frac{m}{n}\right)+(1-\theta) \frac{1}{q}, \text { for all } \theta \text { in the interval } \frac{j}{m} \leq \theta \leq 1
$$

(the constant $c$ depending only on $n, m, j, q, r, \theta)$, with the following exceptional cases

(i) If $j=0, r m<n$ and $q=\infty$, then we make the additional assumption that either $u$ tends to zero at infinity or $u \in L^{\tilde{q}}$ for some finite $\tilde{q}>0$.

(ii) If $1<r<\infty$ and $m-j-n / r$ is a nonnegative integer, then (2.2) holds only for $\theta$ satisfying $j / m \leq \theta<1$.

We will use this simple version of the classical Gronwall Lemma:

Lemma 2.3. Assume $\lambda>0$. Let $y(t)$ and $h(t)$ be two nonnegative functions satisfying

$$
y^{\prime}(t)+\lambda y(t) \leq h(t), \quad \text { for all } t \geq t_{0} \geq 0 .
$$

Then, one has

$$
y(t) \leq e^{\lambda\left(t_{0}-t\right)} y\left(t_{0}\right)+\int_{t_{0}}^{t} e^{\lambda(s-t)} h(s) d s
$$


The proof is straightforward so we omit it here.

In the discussions below, we denote by $\|\cdot\|$ and $(\cdot, \cdot)$ the norm and the inner product in $L^{2}\left(\mathbb{R}^{n}\right)$ and use $\|\cdot\|_{p}$ to denote the norm in $L^{p}\left(\mathbb{R}^{n}\right)$. Also, the letters $c$ and $c_{j}(j=1,2, \cdots)$ are generic positive constants which may change their values from line to line or even in the same line.

\section{Stochastic semilinear fractional power dissipative equation}

In the sequel, we consider the probability space $(\Omega, \mathcal{F}, \mathbb{P})$ where

$$
\Omega=\left\{\omega=\left(\omega_{1}, \omega_{2}, \ldots, \omega_{m}\right) \in C\left(\mathbb{R}, \mathbb{R}^{m}\right): \omega(0)=0\right\}
$$

$\mathcal{F}$ is the Borel $\sigma$-algebra induced by the compact-open topology on $\Omega$, and $\mathbb{P}$ is the corresponding Wiener measure on $(\Omega, \mathcal{F})$. Define a shift on $\omega$ by

$$
\theta_{t} \omega(\cdot)=\omega(\cdot+t)-\omega(t), \quad \omega \in \Omega, \quad t \in \mathbb{R}
$$

Then $\left(\Omega, \mathcal{F}, \mathbb{P},\left(\theta_{t}\right)_{t \in \mathbb{R}}\right)$ is a metric dynamical system.

In this section, we discuss the existence of a continuous random dynamical system representing the stochastic semilinear fractional power equation perturbed by additive noise. Thanks to the special linear additive noise, the stochastic semilinear fractional power equation can be reduced to an equation with random coefficients by a suitable change of variable. To this end, with $\lambda>0$ as before, we consider the stationary process

$$
z_{j}(t)=z_{j}\left(\theta_{t} \omega_{j}\right)=-\lambda \int_{-\infty}^{0} e^{\lambda \tau}\left(\theta_{t} \omega_{j}\right)(\tau) d \tau, \quad t \in \mathbb{R}
$$

which satisfies the stochastic differential equation:

$$
d z_{j}+\lambda z_{j} d t=d \omega_{j}(t)
$$

We know from [1] that there is a $\theta_{t}$-invariant set $\tilde{\Omega} \subset \Omega$ of full measure such that the random variable $\left|z_{j}\left(\omega_{j}\right)\right|$ is tempered and $z_{j}\left(\theta_{t} \omega_{j}\right)$ is continuous for all $\omega \in \tilde{\Omega}$. Therefore, from Proposition 4.3.3 in [1], there exists a tempered function $r(\omega)>0$ such that

$$
\sum_{j=1}^{m}\left(\left|z_{j}\left(\omega_{j}\right)\right|^{2}+\left|z_{j}\left(\omega_{j}\right)\right|^{p}\right) \leq r(\omega),
$$

where $r(\omega)$ satisfies, for $\mathbb{P}$-a.e. $\omega \in \Omega$,

$$
r\left(\theta_{t} \omega\right) \leq e^{\frac{\lambda}{2}|t|} r(\omega), \quad t \in \mathbb{R}
$$


From (3.2) and (3.3), we obtain that, for $\mathbb{P}$-a.e. $\omega \in \Omega$,

$$
\sum_{j=1}^{m}\left(\left|z_{j}\left(\omega_{j}\right)\right|^{2}+\left|z_{j}\left(\omega_{j}\right)\right|^{p}\right) \leq e^{\frac{\lambda}{2}|t|} r(\omega), \quad t \in \mathbb{R} .
$$

Setting $z\left(\theta_{t} \omega\right)=\sum_{j=1}^{m} \phi_{j} z_{j}\left(\theta_{t} \omega_{j}\right)$ and applying (3.1), one has

$$
d z+\lambda z d t=\sum_{j=1}^{m} \phi_{j} d \omega_{j}
$$

We write the unknown $u(t)$ in (1.2) - (1.3) as $u(t)=v(t)+z\left(\theta_{t} \omega\right)$ to obtain the following random differential equation for $v$

$$
v_{t}+(-\triangle)^{\alpha} v+\lambda v=F\left(x, v+z\left(\theta_{t} \omega\right)\right)+(-\triangle)^{\alpha} z\left(\theta_{t} \omega\right)
$$

with the initial data

$$
v(x, 0)=v_{0}(x)=u_{0}(x)-z(\omega), \quad x \in \mathbb{R}^{n} .
$$

Next, we construct a random dynamical system modeling the stochastic fractional power dissipative equation. The existence and uniqueness of the solution to problem (3.5)-(3.6), for a.a. $\omega \in \Omega$, can be obtained (see [19]), and this defines a random dynamical system $(\varphi(t))_{t \geq 0}$ over $\left(\Omega, \mathcal{F}, \mathbb{P},\left(\theta_{t}\right)_{t \in \mathbb{R}}\right)$ by

$$
\varphi\left(t, \omega, v_{0}\right)=v\left(t, \omega, v_{0}\right), \text { for } v_{0} \in L^{2}\left(\mathbb{R}^{n}\right), t \geq 0 \text { and for a.a. } \omega \in \Omega
$$

We now define a mapping $\phi: \mathbb{R}^{+} \times \Omega \times L^{2}\left(\mathbb{R}^{n}\right) \rightarrow L^{2}\left(\mathbb{R}^{n}\right)$ by

$$
\phi\left(t, \omega, u_{0}\right)=u\left(t, \omega, u_{0}\right)=v\left(t, \omega, u_{0}-z(\omega)\right)+z\left(\theta_{t} \omega\right)
$$

for $v_{0} \in L^{2}\left(\mathbb{R}^{n}\right), t \geq 0$ and for a. a. $\omega \in \Omega$. Then $\phi$ is a continuous random dynamical system associated with the problem (3.5) on $L^{2}\left(\mathbb{R}^{n}\right)$. From now on, we replace $\Omega$ by $\tilde{\Omega}$ but still call the space $\Omega$.

Note that the two random dynamical systems $\phi$ and $\varphi$ are equivalent. It is easy to check that $\phi$ has a random attractor provided $\varphi$ possesses a random attractor. Therefore, we only need to consider the random dynamical system $\varphi$. 


\section{Uniform estimates of solutions}

Here, we derive uniform estimates on the solutions of the stochastic fractional power dissipative equation (1.2)-(1.3) on $\mathbb{R}^{n}$ when $t \rightarrow \infty$. These estimates are necessary for proving the existence of bounded absorbing sets and asymptotic compactness of orbits of the random dynamical system associated with the equation. In particular, we will show that the solutions for large space variables are uniformly small when time is sufficiently large.

From now on, we always suppose that $\mathcal{D}$ is the collection of all tempered random subsets of $L^{2}\left(\mathbb{R}^{n}\right)$. First, we derive the following uniform estimates on $u$ in $\mathcal{D}$.

Lemma 4.1. Suppose that (1.4) and (1.5) hold. Let $B=\{B(\omega)\} \in \mathcal{D}$ and $u_{0}(\omega) \in B(\omega)$. Then, for $\omega \in \Omega$, there exists $T_{0_{B}}(\omega)>0$ such that for any $t \geq T_{0_{B}}(\omega)$, one has

$$
\left\|u\left(t, \theta_{-t} \omega, v_{0}\left(\theta_{-t} \omega\right)\right)\right\|^{2} \leq 2\left(c_{8} r(\omega)+c_{8}+\|z(\omega)\|^{2}\right) .
$$

Proof. Taking the inner product in $L^{2}$ of (3.5) with $v$, we obtain

$$
\frac{1}{2} \frac{d}{d t}\|v\|^{2}+\left\|(-\triangle)^{\frac{\alpha}{2}} v\right\|^{2}+\lambda\|v\|^{2}=\int_{\mathbb{R}^{n}} F\left(x, v+z\left(\theta_{t} \omega\right)\right) v d x+\left((-\triangle)^{\alpha} z\left(\theta_{t} \omega\right), v\right) .
$$

Now we estimate the two terms on the right-hand side of (4.8). For the first term, applying conditions (1.4) and (1.5), together with Young's inequality, we obtain

$$
\begin{array}{rl}
\int_{\mathbb{R}^{n}} & F\left(x, v+z\left(\theta_{t} \omega\right)\right) v d x \\
& =\int_{\mathbb{R}^{n}} F\left(x, v+z\left(\theta_{t} \omega\right)\right)\left(v+z\left(\theta_{t} \omega\right)\right) d x-\int_{\mathbb{R}^{n}} F\left(x, v+z\left(\theta_{t} \omega\right)\right) z\left(\theta_{t} \omega\right) d x \\
& \leq-\beta_{1} \int_{\mathbb{R}^{n}}|u|^{p} d x+\int_{\mathbb{R}^{n}} \gamma_{1}(x) d x-\int_{\mathbb{R}^{n}} F\left(x, v+z\left(\theta_{t} \omega\right)\right) z\left(\theta_{t} \omega\right) d x \\
& \leq-\beta_{1} \int_{\mathbb{R}^{n}}|u|^{p} d x+\int_{\mathbb{R}^{n}} \gamma_{1}(x) d x+\beta_{2} \int_{\mathbb{R}^{n}}|u|^{p-1}\left|z\left(\theta_{t} \omega\right)\right| d x+\int_{\mathbb{R}^{n}}\left|\gamma_{2}(x) \| z\left(\theta_{t} \omega\right)\right| d x \\
& \leq-\beta_{1}\|u\|_{p}^{p}+\left\|\gamma_{1}\right\|_{1}+\frac{1}{2} \beta_{1}\|u\|_{p}^{p}+c_{1}\left\|z\left(\theta_{t} \omega\right)\right\|_{p}^{p}+\frac{1}{2}\left\|\gamma_{2}\right\|^{2}+\frac{1}{2}\left\|z\left(\theta_{t} \omega\right)\right\|^{2} \\
& \leq-\frac{1}{2} \beta_{1}\|u\|_{p}^{p}+c_{2}\left(\left\|z\left(\theta_{t} \omega\right)\right\|_{p}^{p}+\left\|z\left(\theta_{t} \omega\right)\right\|^{2}\right)+c_{3} .
\end{array}
$$

For the second term, integrating by parts and applying Young's inequality, we have

$$
\left((-\triangle)^{\alpha} z\left(\theta_{t} \omega\right), v\right)=\left((-\triangle)^{\frac{\alpha}{2}} z\left(\theta_{t} \omega\right),(-\triangle)^{\frac{\alpha}{2}} v\right) \leq \frac{1}{2}\left\|(-\triangle)^{\frac{\alpha}{2}} z\left(\theta_{t} \omega\right)\right\|^{2}+\frac{1}{2}\left\|(-\triangle)^{\frac{\alpha}{2}} v\right\|^{2} .
$$

Substituting (4.9) and (4.10) into (4.8), we get

$$
\begin{aligned}
\frac{d}{d t}\|v\|^{2}+\left\|(-\triangle)^{\frac{\alpha}{2}} v\right\|^{2}+ & \lambda\|v\|^{2}+\beta_{1}\|u\|_{p}^{p} \\
& \leq c_{4}\left(\left\|z\left(\theta_{t} \omega\right)\right\|_{p}^{p}+\left\|z\left(\theta_{t} \omega\right)\right\|^{2}+\left\|(-\triangle)^{\frac{\alpha}{2}} z\left(\theta_{t} \omega\right)\right\|^{2}\right)+c_{5} .
\end{aligned}
$$


With $z\left(\theta_{t} \omega\right)=\sum_{j=1}^{m} \phi_{j} z_{j}\left(\theta_{t} \omega_{j}\right)$ and $\phi_{j} \in H^{2}\left(\mathbb{R}^{n}\right) \cap W^{2, p}\left(\mathbb{R}^{n}\right)$, the right-hand side of (4.11) is bounded by

$$
c_{6} \sum_{j=1}^{m}\left(\left|z_{j}\left(\theta_{t} \omega_{j}\right)\right|^{p}+\left|z_{j}\left(\theta_{t} \omega_{j}\right)\right|^{2}\right)+c_{7} \triangleq \eta_{1}\left(\theta_{t} \omega\right)+c_{7} .
$$

Hence, (4.11) can be rewritten as

$$
\frac{d}{d t}\|v\|^{2}+\left\|(-\triangle)^{\frac{\alpha}{2}} v\right\|^{2}+\lambda\|v\|^{2}+\beta_{1}\|u\|_{p}^{p} \leq \eta_{1}\left(\theta_{t} \omega\right)+c_{7}
$$

which implies, for all $t \geq 0$,

$$
\frac{d}{d t}\|v\|^{2}+\lambda\|v\|^{2} \leq \eta_{1}\left(\theta_{t} \omega\right)+c_{7}
$$

By Lemma 2.3, one has, for all $t \geq 0$,

$$
\| v\left(t, \omega, v_{0}(\omega)\left\|^{2} \leq e^{-\lambda t}\right\| v_{0}(\omega) \|^{2}+\int_{0}^{t} e^{\lambda(s-t)} \eta_{1}\left(\theta_{s} \omega\right) d s+\frac{c_{7}}{\lambda} .\right.
$$

By (3.2) and (3.3), we obtain that for all $\omega \in \Omega$,

$$
\eta_{1}\left(\theta_{s} \omega\right) \leq c_{6} e^{\frac{1}{2} \lambda|s|} r(\omega)
$$

Replacing $\omega$ by $\theta_{-t} \omega$ in (4.15) and using (4.16), we have, for all $t \geq 0$,

$$
\begin{aligned}
\left\|v\left(t, \theta_{-t} \omega, v_{0}\left(\theta_{-t} \omega\right)\right)\right\|^{2} & \leq e^{-\lambda t}\left\|v_{0}\left(\theta_{-t} \omega\right)\right\|^{2}+\int_{0}^{t} e^{\lambda(s-t)} \eta_{1}\left(\theta_{s-t} \omega\right) d s+\frac{c_{7}}{\lambda} \\
& \leq e^{-\lambda t}\left\|v_{0}\left(\theta_{-t} \omega\right)\right\|^{2}+\int_{-t}^{0} e^{\lambda \tau} \eta_{1}\left(\theta_{\tau} \omega\right) d \tau+\frac{c_{7}}{\lambda} \\
& \leq e^{-\lambda t}\left\|v_{0}\left(\theta_{-t} \omega\right)\right\|^{2}+c_{6} \int_{-t}^{0} e^{\frac{1}{2} \lambda \tau} r(\omega) d \tau+\frac{c_{7}}{\lambda} \\
& \leq e^{-\lambda t}\left\|v_{0}\left(\theta_{-t} \omega\right)\right\|^{2}+\frac{2 c_{6}}{\lambda} r(\omega)+\frac{c_{7}}{\lambda}
\end{aligned}
$$

Note that

$$
\phi\left(t, \omega, u_{0}(\omega)\right)=v\left(t, \omega, u_{0}(\omega)-z(\omega)\right)+z\left(\theta_{t} \omega\right) .
$$

It follows from (4.17) that, for all $t \geq 0$,

$$
\begin{aligned}
\left\|\phi\left(t, \theta_{-t} \omega, u_{0}\left(\theta_{-t} \omega\right)\right)\right\|^{2} & =\left\|v\left(t, \theta_{-t} \omega, u_{0}\left(\theta_{-t} \omega\right)\right)+z(\omega)\right\|^{2} \\
& \leq 2\left\|v\left(t, \theta_{-t} \omega, u_{0}\left(\theta_{-t} \omega\right)\right)\right\|^{2}+2\|z(\omega)\|^{2} \\
& \leq 2 e^{-\lambda t}\left\|u_{0}\left(\theta_{-t} \omega\right)-z\left(\theta_{-t} \omega\right)\right\|^{2}+c_{8}(r(\omega)+1)+2\|z(\omega)\|^{2} \\
& \leq 4 e^{-\lambda t}\left(\left\|u_{0}\left(\theta_{-t} \omega\right)\right\|^{2}+\left\|z\left(\theta_{-t} \omega\right)\right\|^{2}\right)+c_{8}(r(\omega)+1)+2\|z(\omega)\|^{2} .
\end{aligned}
$$


By the assumption, $\{B(\omega)\}_{\omega \in \Omega} \in \mathcal{D}$ is tempered. On the other hand, by Definition 2.3, $\|z(\omega)\|^{2}$ is also tempered. Therefore, if $u_{0}\left(\theta_{-t} \omega\right) \in B\left(\theta_{-t} \omega\right)$, then there exists $T_{0_{B}}(\omega)>0$ such that for all $t \geq T_{0_{B}}(\omega)$,

$$
4 e^{-\lambda t}\left(\left\|u_{0}\left(\theta_{-t} \omega\right)\right\|^{2}+\left\|z\left(\theta_{-t} \omega\right)\right\|^{2}\right) \leq c_{8}(r(\omega)+1) .
$$

It follows from (4.18) that, for all $t \geq T_{0_{B}}(\omega)$,

$$
\left\|\phi\left(t, \theta_{-t} \omega, u_{0}\left(\theta_{-t} \omega\right)\right)\right\|^{2} \leq 2\left(c_{8} r(\omega)+c_{8}+\|z(\omega)\|^{2}\right)
$$

The proof is complete.

Next, we deduce the uniform estimates for $v$ in $H^{1}\left(\mathbb{R}^{n}\right)$ and for $u$ in $L^{p}\left(\mathbb{R}^{n}\right)$. Firstly, we have

Lemma 4.2. Suppose that (1.4) and (1.5) hold. Let $B=\{B(\omega)\} \in \mathcal{D}$ and $u_{0}(\omega) \in B(\omega)$. Then, for every $T_{1} \geq 0$ and $\omega \in \Omega$, one has, for all $t \geq T_{1}$,

- the solution $v\left(t, \omega, v_{0}(\omega)\right)$ of problem (3.5)-(3.6) satisfies

$$
\int_{T_{1}}^{t} e^{\lambda(s-t)}\left\|(-\triangle)^{\frac{\alpha}{2}} v\left(s, \theta_{-t} \omega, v_{0}\left(\theta_{-t} \omega\right)\right)\right\|^{2} d s \leq e^{-\lambda t}\left\|v_{0}\left(\theta_{-t} \omega\right)\right\|^{2}+c(r(\omega)+1),
$$

- the solution $u\left(t, \omega, u_{0}(\omega)\right)$ of problem (1.2)-(1.3) satisfies

$$
\beta_{1} \int_{T_{1}}^{t} e^{\lambda(s-t)}\left\|u\left(s, \theta_{-t} \omega, u_{0}\left(\theta_{-t} \omega\right)\right)\right\|_{p}^{p} d s \leq e^{-\lambda t}\left\|v_{0}\left(\theta_{-t} \omega\right)\right\|^{2}+c(r(\omega)+1) .
$$

Proof. Replacing t by $T_{1}$ and then replacing $\omega$ by $\theta_{-t} \omega$ in (4.15), we have

$$
\left\|v\left(T_{1}, \theta_{-t} \omega, v_{0}\left(\theta_{-t} \omega\right)\right)\right\|^{2} \leq e^{-\lambda T_{1}}\left\|v_{0}\left(\theta_{-t} \omega\right)\right\|^{2}+\int_{0}^{T_{1}} e^{\lambda\left(s-T_{1}\right)} \eta_{1}\left(\theta_{s-t} \omega\right) d s+c .
$$

Multiplying the above inequality by $e^{\lambda\left(T_{1}-t\right)}$ and using (4.16), we obtain

$$
\begin{aligned}
e^{\lambda\left(T_{1}-t\right)} \| & v\left(T_{1}, \theta_{-t} \omega, v_{0}\left(\theta_{-t} \omega\right)\right) \|^{2} \\
& \leq e^{-\lambda t}\left\|v_{0}\left(\theta_{-t} \omega\right)\right\|^{2}+\int_{0}^{T_{1}} e^{\lambda(s-t)} \eta_{1}\left(\theta_{s-t} \omega\right) d s+c e^{\lambda\left(T_{1}-t\right)} \\
& =e^{-\lambda t}\left\|v_{0}\left(\theta_{-t} \omega\right)\right\|^{2}+\int_{-t}^{T_{1}-t} e^{\lambda s} \eta_{1}\left(\theta_{s} \omega\right) d s+c e^{\lambda\left(T_{1}-t\right)} \\
& \leq e^{-\lambda t}\left\|v_{0}\left(\theta_{-t} \omega\right)\right\|^{2}+c_{6} r(\omega) \int_{-t}^{T_{1}-t} e^{\frac{1}{2} \lambda s} d s+c e^{\lambda\left(T_{1}-t\right)} \\
& \leq e^{-\lambda t}\left\|v_{0}\left(\theta_{-t} \omega\right)\right\|^{2}+\frac{2}{\lambda} c_{6} r(\omega) e^{\frac{1}{2} \lambda\left(T_{1}-t\right)}+c e^{\lambda\left(T_{1}-t\right)} .
\end{aligned}
$$


By (4.13), one has, for all $t \geq T_{1}$,

$$
\begin{aligned}
\left\|v\left(t, \omega, v_{0}(\omega)\right)\right\|^{2} & +\int_{T_{1}}^{t} e^{\lambda(s-t)}\left\|(-\triangle)^{\frac{\alpha}{2}} v\left(s, \omega, v_{0}(\omega)\right)\right\|^{2} d s+\beta_{1} \int_{T_{1}}^{t} e^{\lambda(s-t)}\left\|u\left(s, \omega, u_{0}(\omega)\right)\right\|_{p}^{p} d s \\
& \leq e^{\lambda\left(T_{1}-t\right)}\left\|v\left(T_{1}, \omega, v_{0}(\omega)\right)\right\|^{2}+\int_{T_{1}}^{t} e^{\lambda(s-t)} \eta_{1}\left(\theta_{s} \omega\right) d s+c \int_{T_{1}}^{t} e^{\lambda(s-t)} d s .
\end{aligned}
$$

Dropping the first term on the left-hand side of (4.22), replacing $\omega$ by $\theta_{-t} \omega$ in (4.15) and using (4.16), we obtain, for all $t \geq T_{1}$,

$$
\begin{aligned}
\int_{T_{1}}^{t} e^{\lambda(s-t)} \| & (-\triangle)^{\frac{\alpha}{2}} v\left(s, \theta_{-t} \omega, v_{0}\left(\theta_{-t} \omega\right)\right)\left\|^{2} d s+\beta_{1} \int_{T_{1}}^{t} e^{\lambda(s-t)}\right\| u\left(s, \theta_{-t} \omega, u_{0}\left(\theta_{-t} \omega\right)\right) \|_{p}^{p} d s \\
& \leq e^{\lambda\left(T_{1}-t\right)}\left\|v\left(T_{1}, \theta_{-t} \omega, v_{0}\left(\theta_{-t} \omega\right)\right)\right\|^{2}+\int_{T_{1}}^{t} e^{\lambda(s-t)} \eta_{1}\left(\theta_{s-t} \omega\right) d s+c \int_{T_{1}}^{t} e^{\lambda(s-t)} d s \\
& \leq e^{\lambda\left(T_{1}-t\right)}\left\|v\left(T_{1}, \theta_{-t} \omega, v_{0}\left(\theta_{-t} \omega\right)\right)\right\|^{2}+\int_{T_{1}-t}^{0} e^{\lambda \tau} \eta_{1}\left(\theta_{\tau} \omega\right) d \tau+\frac{c}{\lambda} \\
& \leq e^{\lambda\left(T_{1}-t\right)}\left\|v\left(T_{1}, \theta_{-t} \omega, v_{0}\left(\theta_{-t} \omega\right)\right)\right\|^{2}+c_{6} r(\omega) \int_{T_{1}-t}^{0} e^{\frac{1}{2} \lambda \tau} d \tau+\frac{c}{\lambda} \\
& \leq e^{\lambda\left(T_{1}-t\right)}\left\|v\left(T_{1}, \theta_{-t} \omega, v_{0}\left(\theta_{-t} \omega\right)\right)\right\|^{2}+\frac{2}{\lambda} c_{6} r(\omega)+\frac{c}{\lambda} \\
& \leq e^{\lambda\left(T_{1}-t\right)}\left\|v\left(T_{1}, \theta_{-t} \omega, v_{0}\left(\theta_{-t} \omega\right)\right)\right\|^{2}+c(r(\omega)+1) .
\end{aligned}
$$

Combining (4.23) and (4.21), we have

$$
\begin{gathered}
\int_{T_{1}}^{t} e^{\lambda(s-t)} \|(-\triangle)^{\frac{\alpha}{2}} \\
v\left(s, \theta_{-t} \omega, v_{0}\left(\theta_{-t} \omega\right)\right)\left\|^{2} d s+\beta_{1} \int_{T_{1}}^{t} e^{\lambda(s-t)}\right\| u\left(s, \theta_{-t} \omega, u_{0}\left(\theta_{-t} \omega\right)\right) \|_{p}^{p} d s \\
\leq e^{-\lambda t}\left\|v_{0}\left(\theta_{-t} \omega\right)\right\|^{2}+c(r(\omega)+1) .
\end{gathered}
$$

This completes the proof.

Lemma 4.3. Suppose that (1.4) and (1.5) hold. Let $B=\{B(\omega)\} \in \mathcal{D}$ and $u_{0}(\omega) \in B(\omega)$. Then, for $\omega \in \Omega$, there exists $T_{0_{B}}(\omega)>0$ such that for all $t \geq T_{0_{B}}(\omega)$,

(i) the solution $u\left(t, \omega, u_{0}(\omega)\right)$ of problem (1.2)-(1.3) satisfies

$$
\begin{aligned}
& \beta_{1} \int_{t}^{t+1}\left\|u\left(s, \theta_{-t-1} \omega, v_{0}\left(\theta_{-t-1} \omega\right)\right)\right\|_{p}^{p} d s \leq 2 e^{\lambda}(r(\omega)+1), \\
& \int_{t}^{t+1}\left\|(-\triangle)^{\frac{\alpha}{2}} u\left(s, \theta_{-t-1} \omega, u_{0}\left(\theta_{-t-1} \omega\right)\right)\right\|^{2} d s \leq c(r(\omega)+1),
\end{aligned}
$$

(ii) the solution $v\left(t, \omega, v_{0}(\omega)\right)$ of problem (3.5)-(3.6) satisfies

$$
\int_{t}^{t+1}\left\|(-\triangle)^{\frac{\alpha}{2}} v\left(s, \theta_{-t-1} \omega, v_{0}\left(\theta_{-t-1} \omega\right)\right)\right\|^{2} d s \leq 2 e^{\lambda}(r(\omega)+1) .
$$


Proof. Replacing $\mathrm{t}$ by $t+1$ and then replacing $T_{1}$ by $t$ in (4.19), we have

$$
\begin{aligned}
\int_{t}^{t+1} e^{\lambda(s-t-1)} \|(-\triangle)^{\frac{\alpha}{2}} v\left(s, \theta_{-t-1} \omega,\right. & \left.v_{0}\left(\theta_{-t-1} \omega\right)\right) \|^{2} d s \\
& \leq e^{-\lambda(t+1)}\left\|v_{0}\left(\theta_{-t-1} \omega\right)\right\|^{2}+c(r(\omega)+1) .
\end{aligned}
$$

For all $s \in[t, t+1]$, we know $e^{\lambda(s-t-1)} \geq e^{-\lambda}$. So, (4.27) can be rewritten as

$$
\begin{aligned}
e^{-\lambda} \int_{t}^{t+1} \|(-\triangle)^{\frac{\alpha}{2}} & v\left(s, \theta_{-t-1} \omega, v_{0}\left(\theta_{-t-1} \omega\right)\right) \|^{2} d s \\
\leq & e^{-\lambda(t+1)}\left\|v_{0}\left(\theta_{-t-1} \omega\right)\right\|^{2}+c(r(\omega)+1) \\
\leq & 2 e^{-\lambda(t+1)}\left(\left\|u_{0}\left(\theta_{-t-1} \omega\right)\right\|^{2}+\left\|z\left(\theta_{-t-1} \omega\right)\right\|^{2}\right)+c(r(\omega)+1)
\end{aligned}
$$

Since $\left\|u_{0}(\omega)\right\|^{2}$ and $\|z(\omega)\|^{2}$ are tempered, there exists $T_{0_{B}}(\omega)>0$ such that for all $t \geq T_{0_{B}}(\omega)$,

$$
2 e^{-\lambda(t+1)}\left(\left\|u_{0}\left(\theta_{-t-1} \omega\right)\right\|^{2}+\left\|z\left(\theta_{-t-1} \omega\right)\right\|^{2}\right) \leq c(r(\omega)+1)
$$

It follows from (4.28) that, for all $t \geq T_{0_{B}}(\omega)$,

$$
\int_{t}^{t+1}\left\|(-\triangle)^{\frac{\alpha}{2}} v\left(s, \theta_{-t-1} \omega, v_{0}\left(\theta_{-t-1} \omega\right)\right)\right\|^{2} d s \leq 2 e^{\lambda}(r(\omega)+1) .
$$

Using (4.20) and repeating the above process, we have, for all $t \geq T_{0_{B}}(\omega)$,

$$
\beta_{1} \int_{t}^{t+1}\left\|u\left(s, \theta_{-t-1} \omega, v_{0}\left(\theta_{-t-1} \omega\right)\right)\right\|_{p}^{p} d s \leq 2 e^{\lambda}(r(\omega)+1) .
$$

By (4.29), one has

$$
\begin{aligned}
\int_{t}^{t+1} & \left\|(-\triangle)^{\frac{\alpha}{2}} u\left(s, \theta_{-t-1} \omega, u_{0}\left(\theta_{-t-1} \omega\right)\right)\right\|^{2} d s \\
& \leq 2 \int_{t}^{t+1}\left\|(-\triangle)^{\frac{\alpha}{2}} v\left(s, \theta_{-t-1} \omega, v_{0}\left(\theta_{-t-1} \omega\right)\right)\right\|^{2} d s+2 \int_{t}^{t+1}\left\|(-\triangle)^{\frac{\alpha}{2}} z\left(\theta_{s-t-1} \omega\right)\right\|^{2} d s \\
& \leq c(r(\omega)+1)+c \int_{t}^{t+1} e^{\frac{\lambda}{2}(t+1-s)} r(\omega) d s \\
& \leq c(r(\omega)+1) .
\end{aligned}
$$

This completes the proof.

Lemma 4.4. Suppose that (1.4)-(1.7) hold. Let $B=\{B(\omega)\} \in \mathcal{D}$ and $u_{0}(\omega) \in B(\omega)$. Then for $\omega \in \Omega$, there exists $T_{0_{B}}(\omega)>0$ such that for all $t \geq T_{0_{B}}(\omega)$, the solution $u\left(t, \omega, u_{0}(\omega)\right)$ of problem (1.2)-(1.3) satisfies,

$$
\left\|(-\triangle)^{\frac{\alpha}{2}} u\left(t+1, \theta_{-t-1} \omega, u_{0}\left(\theta_{-t-1} \omega\right)\right)\right\|^{2} \leq c(r(\omega)+1) .
$$


Proof. Taking the inner product in $L^{2}$ of (3.5) with $(-\triangle)^{\alpha} v$, we obtain

$$
\begin{aligned}
\frac{1}{2} \frac{d}{d t}\left\|(-\triangle)^{\frac{\alpha}{2}} v\right\|^{2} & +\lambda\left\|(-\triangle)^{\frac{\alpha}{2}} v\right\|^{2}+\left\|(-\triangle)^{\alpha} v\right\|^{2} \\
& =\int_{\mathbb{R}^{n}} F(x, u)(-\triangle)^{\alpha} v d x+\left((-\triangle)^{\alpha} z\left(\theta_{t} \omega\right),(-\triangle)^{\alpha} v\right)
\end{aligned}
$$

Now, we will estimate the first term on the right-hand side of (4.33). Integrating by parts, using (1.6) and (1.7), then using Young's inequality and the Gagliardo-Nirenberg inequality, we infer that

$$
\begin{array}{rl}
\int_{\mathbb{R}^{n}} & F(x, u)(-\triangle)^{\alpha} v d x \\
& \leq \int_{\mathbb{R}^{n}}\left|\frac{\partial F}{\partial x}(x, u)\right|\left|(-\triangle)^{\alpha-\frac{1}{2}} v\right| d x+\int_{\mathbb{R}^{n}}\left|\frac{\partial F}{\partial u}(x, u)\right||\nabla u|\left|(-\triangle)^{\alpha-\frac{1}{2}} v\right| d x \\
& \leq\left\|\gamma_{3}\right\|\left\|(-\triangle)^{\alpha-\frac{1}{2}} v\right\|+\beta_{3}\|\nabla u\|\left\|(-\triangle)^{\alpha-\frac{1}{2}} v\right\| \\
& \leq \frac{1}{2}\left(\left\|\gamma_{3}\right\|^{2}+\beta^{2}\left(\|\nabla v\|^{2}+\left\|\nabla z\left(\theta_{t} \omega\right)\right\|^{2}\right)+\left\|(-\triangle)^{\alpha-\frac{1}{2}} v\right\|^{2}\right) \\
& \leq \frac{1}{2}\left(\left\|\gamma_{3}\right\|^{2}+\beta^{2}\left\|\nabla z\left(\theta_{t} \omega\right)\right\|^{2}+c \beta^{2}\left\|(-\triangle)^{\alpha} v\right\|^{\frac{1}{\alpha}}\|v\|^{\frac{2 \alpha-1}{\alpha}}+c\left\|(-\triangle)^{\alpha} v\right\|^{\frac{2 \alpha-1}{\alpha}}\|v\|^{\frac{1}{\alpha}}\right) \\
& \leq \frac{1}{4}\left\|(-\triangle)^{\alpha} v\right\|^{2}+c\left(\left\|\gamma_{3}\right\|^{2}+\|u\|^{2}+\left\|z\left(\theta_{t} \omega\right)\right\|^{2}+\left\|\nabla z\left(\theta_{t} \omega\right)\right\|^{2}\right)
\end{array}
$$

and

$$
\begin{aligned}
\left((-\triangle)^{\alpha} z\left(\theta_{t} \omega\right),(-\triangle)^{\alpha} v\right) & \leq\left\|(-\triangle)^{\alpha} z\left(\theta_{t} \omega\right)\right\|\left\|(-\triangle)^{\alpha} v\right\| \\
& \leq \frac{1}{4}\left\|(-\triangle)^{\alpha} v\right\|^{2}+c\left(\left\|\triangle z\left(\theta_{t} \omega\right)\right\|^{2}+\left\|z\left(\theta_{t} \omega\right)\right\|^{2}\right) .
\end{aligned}
$$

Substituting (4.34) and (4.35) into (4.33), we infer that

$$
\frac{d}{d t}\left\|(-\triangle)^{\frac{\alpha}{2}} v\right\|^{2}+2 \lambda\left\|(-\triangle)^{\frac{\alpha}{2}} v\right\|^{2}+\left\|(-\triangle)^{\alpha} v\right\|^{2} \leq c\|u\|^{2}+\eta_{2}\left(\theta_{t} \omega\right)
$$

where $\eta_{2}\left(\theta_{t} \omega\right)=c\left(\left\|\triangle z\left(\theta_{t} \omega\right)\right\|^{2}+\left\|\nabla z\left(\theta_{t} \omega\right)\right\|^{2}+\left\|z\left(\theta_{t} \omega\right)\right\|^{2}+c\right)$.

Note that $z\left(\theta_{t} \omega\right)=\sum_{j=1}^{m} \phi_{j} z_{j}\left(\theta_{t} \omega_{j}\right)$ and $\phi_{j} \in H^{2}\left(\mathbb{R}^{n}\right) \cap W^{2, p}\left(\mathbb{R}^{n}\right)$, together with (3.3), we obtain

$$
\eta_{2}\left(\theta_{t} \omega\right) \leq c \sum_{j=1}^{m}\left|z_{j}\left(\theta_{t} \omega_{j}\right)\right|^{2}+c \leq c e^{\frac{\lambda}{2}|t|} r(\omega)+c, \text { for all } t \in \mathbb{R} .
$$

Let $T_{0_{B}}(\omega)$ be the positive constant in Lemma 4.3, take $t \geq T_{0_{B}}(\omega)$ and $s \in(t, t+1)$. Integrating 
(4.36) over $(s, t+1)$, one has

$$
\begin{aligned}
\left\|(-\triangle)^{\frac{\alpha}{2}} v\left(t+1, \omega, v_{0}(\omega)\right)\right\|^{2} \leq & \left\|(-\triangle)^{\frac{\alpha}{2}} v\left(s, \omega, v_{0}(\omega)\right)\right\|^{2}+c \int_{s}^{t+1} \| u\left(\tau, \omega, u_{0}(\omega) \|^{2} d \tau\right. \\
& +\int_{s}^{t+1} \eta_{2}\left(\theta_{\tau} \omega\right) d \tau \\
\leq & \left\|(-\triangle)^{\frac{\alpha}{2}} v\left(s, \omega, v_{0}(\omega)\right)\right\|^{2}+c \int_{t}^{t+1} \| u\left(\tau, \omega, u_{0}(\omega) \|^{2} d \tau\right. \\
& +\int_{t}^{t+1} \eta_{2}\left(\theta_{\tau} \omega\right) d \tau .
\end{aligned}
$$

Now integrating the above inequality with respect to s over $(t, t+1)$, we infer that

$$
\begin{aligned}
\left\|(-\triangle)^{\frac{\alpha}{2}} v\left(t+1, \omega, v_{0}(\omega)\right)\right\|^{2} \leq & \int_{t}^{t+1}\left\|(-\triangle)^{\frac{\alpha}{2}} v\left(s, \omega, v_{0}(\omega)\right)\right\|^{2} d s \\
& +c \int_{t}^{t+1} \| u\left(\tau, \omega, u_{0}(\omega) \|^{2} d \tau+\int_{t}^{t+1} \eta_{2}\left(\theta_{\tau} \omega\right) d \tau .\right.
\end{aligned}
$$

Replacing $\omega$ by $\theta_{-t-1} \omega$, we deduce that

$$
\begin{gathered}
\left\|(-\triangle)^{\frac{\alpha}{2}} v\left(t+1, \theta_{-t-1} \omega, v_{0}\left(\theta_{-t-1} \omega\right)\right)\right\|^{2} \leq \int_{t}^{t+1}\left\|(-\triangle)^{\frac{\alpha}{2}} v\left(s, \theta_{-t-1} \omega, v_{0}\left(\theta_{-t-1} \omega\right)\right)\right\|^{2} d s \\
+c \int_{t}^{t+1} \| u\left(\tau, \theta_{-t-1} \omega, u_{0}\left(\theta_{-t-1} \omega\right) \|^{2} d \tau+\int_{t}^{t+1} \eta_{2}\left(\theta_{\tau-t-1} \omega\right) d \tau .\right.
\end{gathered}
$$

It follows from (4.26) and (4.24) that, for all $t \geq T_{0_{B}}(\omega)$,

$$
\begin{gathered}
\left\|(-\triangle)^{\frac{\alpha}{2}} v\left(t+1, \theta_{-t-1} \omega, v_{0}\left(\theta_{-t-1} \omega\right)\right)\right\|^{2} \leq c(r(\omega)+1)+\int_{-1}^{0} \eta_{2}\left(\theta_{\tau} \omega\right) d \tau \\
\leq c(r(\omega)+1)+\int_{-1}^{0}\left(c e^{-\frac{\lambda}{2} \tau} r(\omega)+c\right) d \tau \\
\leq c(r(\omega)+1) .
\end{gathered}
$$

By (3.3) and (4.39), we obtain that, for all $t \geq T_{0_{B}}(\omega)$,

$$
\begin{aligned}
\|(-\triangle)^{\frac{\alpha}{2}} u\left(t+1, \theta_{-t-1} \omega,\right. & \left.u_{0}\left(\theta_{-t-1} \omega\right)\right)\left\|^{2}=\right\|(-\triangle)^{\frac{\alpha}{2}} v\left(t+1, \theta_{-t-1} \omega, v_{0}\left(\theta_{-t-1} \omega\right)\right)+(-\triangle)^{\frac{\alpha}{2}} z(\omega) \|^{2} \\
& \leq 2\left(\left\|(-\triangle)^{\frac{\alpha}{2}} v\left(t+1, \theta_{-t-1} \omega, v_{0}\left(\theta_{-t-1} \omega\right)\right)\right\|^{2}+\left\|(-\triangle)^{\frac{\alpha}{2}} z(\omega)\right\|^{2}\right) \\
\leq & c(r(\omega)+1)
\end{aligned}
$$

which completes the proof.

Following from Lemma 4.4, we obtain

Lemma 4.5. Suppose that (1.4)-(1.7) hold. Let $B=\{B(\omega)\} \in \mathcal{D}$ and $u_{0}(\omega) \in B(\omega)$. Then, for $\omega \in \Omega$, there exists $T_{0_{B}}(\omega)>0$ such that for all $t \geq T_{0_{B}}(\omega)+1$, 
(i) the solution $u\left(t, \omega, u_{0}(\omega)\right)$ of problem (1.2)-(1.3) satisfies

$$
\int_{t}^{t+1}\left\|(-\triangle)^{\alpha} u\left(s, \theta_{-t-1} \omega, u_{0}\left(\theta_{-t-1} \omega\right)\right)\right\|^{2} d s \leq c(r(\omega)+1),
$$

(ii) the solution $v\left(t, \omega, v_{0}(\omega)\right)$ of problem (3.5)-(3.6) satisfies

$$
\int_{t}^{t+1}\left\|(-\triangle)^{\alpha} v\left(s, \theta_{-t-1} \omega, v_{0}\left(\theta_{-t-1} \omega\right)\right)\right\|^{2} d s \leq c(r(\omega)+1) .
$$

Proof. From (4.36), it follows that

$$
\frac{d}{d t}\left(e^{\lambda t}\left\|(-\triangle)^{\frac{\alpha}{2}} v\right\|^{2}\right)+e^{\lambda t}\left\|(-\triangle)^{\alpha} v\right\|^{2} \leq c e^{\lambda t}\|u\|^{2}+e^{\lambda t} \eta_{2}\left(\theta_{t} \omega\right)
$$

Integrating (4.42) over $(s, t)\left(t>s \geq T_{0_{B}}(\omega)+1\right)$, then multiplying $e^{-\lambda t}$, one has

$$
\begin{gathered}
\left\|(-\triangle)^{\frac{\alpha}{2}} v\left(t, \omega, v_{0}(\omega)\right)\right\|^{2} \leq e^{\lambda(s-t)}\left\|(-\triangle)^{\frac{\alpha}{2}} v\left(s, \omega, v_{0}(\omega)\right)\right\|^{2}+c \int_{s}^{t} e^{\lambda(\tau-t)} \| u\left(\tau, \omega, u_{0}(\omega) \|^{2} d \tau\right. \\
+\int_{s}^{t} e^{\lambda(\tau-t)} \eta_{2}\left(\theta_{\tau} \omega\right) d \tau
\end{gathered}
$$

Replacing $t$ by $T_{2}$ and $\omega$ by $\theta_{-s} \omega$, we deduce that

$$
\begin{aligned}
\left\|(-\triangle)^{\frac{\alpha}{2}} v\left(T_{2}, \theta_{-s} \omega, v_{0}\left(\theta_{-s} \omega\right)\right)\right\|^{2} \leq & e^{\lambda\left(s-T_{2}\right)}\left\|(-\triangle)^{\frac{\alpha}{2}} v\left(s, \theta_{-s} \omega, v_{0}\left(\theta_{-s} \omega\right)\right)\right\|^{2} \\
& +c \int_{s}^{T_{2}} e^{\lambda\left(\tau-T_{2}\right)} \| u\left(\tau, \theta_{-s} \omega, u_{0}\left(\theta_{-s} \omega\right) \|^{2} d \tau\right. \\
& +\int_{s}^{T_{2}} e^{\lambda\left(\tau-T_{2}\right)} \eta_{2}\left(\theta_{\tau-s} \omega\right) d \tau .
\end{aligned}
$$

From (4.39), we obtain that for $s \geq T_{0_{B}}(\omega)+1$,

$$
e^{\lambda\left(s-T_{2}\right)}\left\|(-\triangle)^{\frac{\alpha}{2}} v\left(s, \theta_{-s} \omega, v_{0}\left(\theta_{-s} \omega\right)\right)\right\|^{2} \leq c(r(\omega)+1) .
$$

Now we estimate the second term on the right-hand side of above inequality. From (4.15), we deduce

$$
\begin{aligned}
\| v\left(\tau, \theta_{-s} \omega, v_{0}\left(\theta_{-s} \omega\right) \|^{2}\right. & \leq e^{-\lambda \tau}\left\|v_{0}\left(\theta_{-s} \omega\right)\right\|^{2}+\int_{0}^{\tau} e^{\lambda(m-\tau)} \eta_{1}\left(\theta_{m-s} \omega\right) d m+\frac{c_{7}}{\lambda} \\
\leq & e^{-\lambda s}\left(\left\|u_{0}\left(\theta_{-s} \omega\right)\right\|^{2}+\left\|z\left(\theta_{-s} \omega\right)\right\|^{2}\right)+\int_{0}^{\tau} e^{\lambda(m-\tau)} \eta_{1}\left(\theta_{m-s} \omega\right) d m+\frac{c_{7}}{\lambda} .
\end{aligned}
$$

Applying (4.16), $u_{0}\left(\theta_{-t} \omega\right) \in B\left(\theta_{-t} \omega\right)$ and $\tau \in\left(s, T_{2}\right)$, we infer that there exists $T_{0_{B}}(\omega)>0$ such that for all $s \geq T_{0_{B}}(\omega)$,

$$
\| v\left(\tau, \theta_{-s} \omega, v_{0}\left(\theta_{-s} \omega\right) \|^{2} \leq c(r(\omega)+1)+c r(\omega) e^{\lambda\left(\tau-\frac{1}{2} s\right)} .\right.
$$


Then we obtain

$$
\begin{aligned}
\| u\left(\tau, \theta_{-s} \omega, v_{0}\left(\theta_{-s} \omega\right) \|^{2}\right. & \leq \| v\left(\tau, \theta_{-s} \omega, v_{0}\left(\theta_{-s} \omega\right)\left\|^{2}+\right\| z(\omega) \|^{2}\right. \\
& \leq c(r(\omega)+1)+c r(\omega) e^{\lambda\left(\tau-\frac{1}{2} s\right)} .
\end{aligned}
$$

So, for $T_{2} \geq 3 / 2 s$, the second term on the right-hand side of (4.43) can be bounded by

$$
\begin{aligned}
\int_{s}^{T_{2}} e^{\lambda\left(\tau-T_{2}\right)} & \| u\left(\tau, \theta_{-s} \omega, v_{0}\left(\theta_{-s} \omega\right) \|^{2} d \tau \leq \int_{s}^{T_{2}} e^{\lambda\left(\tau-T_{2}\right)}\left(c(r(\omega)+1)+c r(\omega) e^{\lambda\left(\tau-\frac{1}{2} s\right)}\right) d \tau\right. \\
& \leq c(r(\omega)+1)\left(1-e^{\lambda\left(s-T_{2}\right)}\right)+\operatorname{cr}(\omega)\left(e^{\frac{1}{2} \lambda s-\lambda T_{2}}-e^{\frac{3}{2} \lambda s-\lambda T_{2}}\right) \\
& \leq c(r(\omega)+1) .
\end{aligned}
$$

Applying (4.37), the third term on the right-hand side of (4.43) can be bounded by

$$
\int_{s}^{T_{2}} e^{\lambda\left(\tau-T_{2}\right)} \eta_{2}\left(\theta_{\tau-s} \omega\right) d \tau \leq \int_{s}^{T_{2}} e^{\lambda\left(\tau-T_{2}\right)}\left(c e^{\frac{\lambda}{2}|\tau-s|}+c\right) d \tau \leq c e^{\lambda T_{2}-\frac{\lambda}{2} s}+c .
$$

Substituting (4.44), (4.48) and (4.49) into (4.43), we obtain

$$
\left\|(-\triangle)^{\frac{\alpha}{2}} v\left(T_{2}, \theta_{-s} \omega, v_{0}\left(\theta_{-s} \omega\right)\right)\right\|^{2} \leq c\left(r(\omega)+e^{\lambda T_{2}-\frac{\lambda}{2} s}+1\right) .
$$

Integrating (4.42) over $\left(T_{2}, t\right)$, one has

$$
\begin{aligned}
\left\|(-\triangle)^{\frac{\alpha}{2}} v\left(t, \omega, v_{0}(\omega)\right)\right\|^{2}+\int_{T_{2}}^{t} e^{\lambda(s-t)}\left\|(-\triangle)^{\alpha} v\left(s, \omega, v_{0}(\omega)\right)\right\|^{2} d s \\
\leq e^{\lambda\left(T_{2}-t\right)}\left\|(-\triangle)^{\frac{\alpha}{2}} v\left(T_{2}, \omega, v_{0}(\omega)\right)\right\|^{2}+c \int_{T_{2}}^{t} e^{\lambda(s-t)}\left\|u\left(s, \omega, v_{0}(\omega)\right)\right\|^{2} d s \\
\quad+\int_{T_{2}}^{t} e^{\lambda(s-t)} \eta_{2}\left(\theta_{s} \omega\right) d s
\end{aligned}
$$

Dropping the first term on the left-side hand of (4.51), and replacing $\omega$ by $\theta_{-t} \omega$, one has

$$
\begin{aligned}
\int_{T_{2}}^{t} e^{\lambda(s-t)} \|( & -\triangle)^{\alpha} v\left(s, \theta_{-t} \omega, v_{0}\left(\theta_{-t} \omega\right)\right)\left\|^{2} d s \leq e^{\lambda\left(T_{2}-t\right)}\right\|(-\triangle)^{\frac{\alpha}{2}} v\left(T_{2}, \theta_{-t} \omega, v_{0}\left(\theta_{-t} \omega\right)\right) \|^{2} \\
& +c \int_{T_{2}}^{t} e^{\lambda(s-t)}\left\|u\left(s, \theta_{-t} \omega, v_{0}\left(\theta_{-t} \omega\right)\right)\right\|^{2} d s+\int_{T_{2}}^{t} e^{\lambda(s-t)} \eta_{2}\left(\theta_{s-t} \omega\right) d s \\
\leq & c\left(r(\omega)+e^{\frac{1}{2} \lambda T_{2}}+1\right)+e^{-\lambda t}\left\|v_{0}\left(\theta_{-t} \omega\right)\right\|^{2}+c(r(\omega)+1)+c\left(1-e^{\frac{\lambda}{2}\left(T_{2}-t\right)}\right) \\
\leq & c(r(\omega)+1) .
\end{aligned}
$$

Replacing $\mathrm{t}$ by $t+1$ and then replacing $T_{2}$ by $t$ in (4.52), we have

$$
\int_{t}^{t+1} e^{\lambda(s-t-1)}\left\|(-\triangle)^{\alpha} v\left(s, \theta_{-t-1} \omega, v_{0}\left(\theta_{-t-1} \omega\right)\right)\right\|^{2} d s \leq c(r(\omega)+1) .
$$


For all $s \in[t, t+1]$, note that $e^{\lambda(s-t-1)} \geq e^{-\lambda}$. Then, (4.53) can be rewritten as

$$
e^{-\lambda} \int_{t}^{t+1}\left\|(-\triangle)^{\alpha} v\left(s, \theta_{-t-1} \omega, v_{0}\left(\theta_{-t-1} \omega\right)\right)\right\|^{2} d s \leq c(r(\omega)+1) .
$$

Therefore, we obtain

$$
\int_{t}^{t+1}\left\|(-\triangle)^{\alpha} v\left(s, \theta_{-t-1} \omega, v_{0}\left(\theta_{-t-1} \omega\right)\right)\right\|^{2} d s \leq c e^{\lambda}(r(\omega)+1) \leq c(r(\omega)+1)
$$

and

$$
\begin{aligned}
\int_{t}^{t+1} & \left\|(-\triangle)^{\alpha} u\left(s, \theta_{-t-1} \omega, u_{0}\left(\theta_{-t-1} \omega\right)\right)\right\|^{2} d s \\
& \leq 2 \int_{t}^{t+1}\left\|(-\triangle)^{\alpha} v\left(s, \theta_{-t-1} \omega, v_{0}\left(\theta_{-t-1} \omega\right)\right)\right\|^{2} d s+2 \int_{t}^{t+1}\left\|(-\triangle)^{\alpha} z\left(\theta_{s-t-1} \omega\right)\right\|^{2} d s \\
& \leq c(r(\omega)+1)+c \int_{t}^{t+1} e^{\frac{\lambda}{2}(t+1-s)} r(\omega) d s \\
& \leq c(r(\omega)+1) .
\end{aligned}
$$

We will also need the following:

Lemma 4.6. Suppose that (1.4)-(1.7) hold. Let $B=\{B(\omega)\} \in \mathcal{D}$ and $u_{0}(\omega) \in B(\omega)$. Then for all $\omega \in \Omega$, there exists $T_{0_{B}}(\omega)>0$ such that for all $t \geq T_{0_{B}}(\omega)+1$, the solution $u\left(t, \omega, u_{0}(\omega)\right)$ of problem (1.2)-(1.3) satisfies

$$
\left\|\nabla u\left(t+1, \theta_{-t-1} \omega, v_{0}\left(\theta_{-t-1} \omega\right)\right)\right\|^{2} \leq c(r(\omega)+1)
$$

Proof. Taking the inner product in $L^{2}$ of (3.5) with $-\Delta v$, we obtain

$$
\frac{1}{2} \frac{d}{d t}\|\nabla v\|^{2}+\lambda\|\nabla v\|^{2}+\left\|(-\triangle)^{\frac{\alpha+1}{2}} v\right\|^{2}=-\int_{\mathbb{R}^{n}} F(x, u) \triangle v d x-\left((-\triangle)^{\alpha} z\left(\theta_{t} \omega\right), \triangle v\right) .
$$

Now, we estimate the first term on the right-hand side of (4.56). Integrating by parts and using (1.5)-(1.7), then by Young's inequality, we infer that

$$
\begin{array}{rl}
-\int_{\mathbb{R}^{n}} & F(x, u) \triangle v d x \\
& =-\int_{\mathbb{R}^{n}} F(x, u) \triangle u d x+\int_{\mathbb{R}^{n}} F(x, u) \triangle z\left(\theta_{t} \omega\right) d x \\
& =\int_{\mathbb{R}^{n}} \frac{\partial F}{\partial x}(x, u) \nabla u d x+\int_{\mathbb{R}^{n}} \frac{\partial F}{\partial u}|\nabla u|^{2} d x+\int_{\mathbb{R}^{n}} F(x, u) \triangle z\left(\theta_{t} \omega\right) d x \\
& \leq\left\|\gamma_{3}\right\|\|\nabla u\|+\beta_{3}\|\nabla u\|^{2}+\int_{\mathbb{R}^{n}}\left|F(x, u) \| \triangle z\left(\theta_{t} \omega\right)\right| d x \\
& \leq\left\|\gamma_{3}\right\|\|\nabla u\|+\beta_{3}\|\nabla u\|^{2}+\beta_{2} \int_{\mathbb{R}^{n}}|u|^{p-1}\left|\triangle z\left(\theta_{t} \omega\right)\right| d x+\int_{\mathbb{R}^{n}}\left|\gamma_{2}(x)\right|\left|\triangle z\left(\theta_{t} \omega\right)\right| d x \\
& \leq c\left(\|\nabla u\|^{2}+\|u\|_{p}^{p}\right)+c\left(\left\|\triangle z\left(\theta_{t} \omega\right)\right\|^{2}+\left\|\triangle z\left(\theta_{t} \omega\right)\right\|_{p}^{p}+1\right) .
\end{array}
$$


For the second term on the right-hand side of (4.56), integrating by parts, then using the GagliardoNirenberg inequality and Young's inequality, one has

$$
\begin{aligned}
\left((-\triangle)^{\alpha} z\left(\theta_{t} \omega\right), \triangle v\right) & =\int_{\mathbb{R}^{n}}(-\triangle)^{\frac{\alpha+1}{2}} z\left(\theta_{t} \omega\right)(-\triangle)^{\frac{\alpha+1}{2}} v d x \\
& \leq \frac{1}{2}\left\|(-\triangle)^{\frac{\alpha+1}{2}} v\right\|^{2}+\frac{1}{2}\left\|(-\triangle)^{\frac{\alpha+1}{2}} z\left(\theta_{t} \omega\right)\right\|^{2} \\
& \leq \frac{1}{2}\left\|(-\triangle)^{\frac{\alpha+1}{2}} v\right\|^{2}+c\left(\left\|\triangle z\left(\theta_{t} \omega\right)\right\|^{2}+\left\|z\left(\theta_{t} \omega\right)\right\|^{2}\right) .
\end{aligned}
$$

Substituting (4.57) and (4.58) into (4.56), we obtain

$$
\frac{d}{d t}\|\nabla v\|^{2}+2 \lambda\|\nabla v\|^{2}+\left\|(-\triangle)^{\frac{\alpha+1}{2}} v\right\|^{2} \leq c\left(\|\nabla u\|^{2}+\|u\|_{p}^{p}\right)+\eta_{3}\left(\theta_{t} \omega\right),
$$

where $\eta_{3}\left(\theta_{t} \omega\right)=c\left(\left\|\triangle z\left(\theta_{t} \omega\right)\right\|^{2}+\left\|\triangle z\left(\theta_{t} \omega\right)\right\|_{p}^{p}+\left\|z\left(\theta_{t} \omega\right)\right\|^{2}+1\right)$. This implies that

$$
\frac{d}{d t}\|\nabla v\|^{2} \leq c\left(\|\nabla u\|^{2}+\|u\|_{p}^{p}\right)+\eta_{3}\left(\theta_{t} \omega\right)
$$

Let $T_{0_{B}}(\omega)$ be the positive constant in Lemma 4.4, take $t \geq T_{0_{B}}(\omega)$ and $s \in(t, t+1)$. Integrating (4.60) over $(s, t+1)$, one has

$$
\begin{aligned}
\left\|\nabla v\left(t+1, \omega, v_{0}(\omega)\right)\right\|^{2} \leq & \left\|\nabla v\left(s, \omega, v_{0}(\omega)\right)\right\|^{2}+\int_{s}^{t+1}\left(\| \nabla u\left(\tau, \omega, v_{0}(\omega)\left\|^{2}+\right\| u\left(\tau, \omega, v_{0}(\omega) \|_{p}^{p}\right) d \tau\right.\right. \\
& +c \int_{s}^{t+1} \eta_{3}\left(\theta_{\tau} \omega\right) d \tau \\
\leq & \left\|\nabla v\left(s, \omega, v_{0}(\omega)\right)\right\|^{2}+\int_{t}^{t+1}\left(\| \nabla u\left(\tau, \omega, v_{0}(\omega)\left\|^{2}+\right\| u\left(\tau, \omega, v_{0}(\omega) \|_{p}^{p}\right) d \tau\right.\right. \\
& +c \int_{t}^{t+1} \eta_{3}\left(\theta_{\tau} \omega\right) d \tau .
\end{aligned}
$$

Now integrating the above with respect to s over $(t, t+1)$, we infer that

$$
\begin{aligned}
\left\|\nabla v\left(t+1, \omega, v_{0}(\omega)\right)\right\|^{2} \leq & \int_{t}^{t+1}\left\|\nabla v\left(s, \omega, v_{0}(\omega)\right)\right\|^{2} d s+\int_{t}^{t+1} \eta_{3}\left(\theta_{\tau} \omega\right) d \tau \\
& +c \int_{t}^{t+1}\left(\| \nabla u\left(\tau, \omega, v_{0}(\omega)\left\|^{2}+\right\| u\left(\tau, \omega, v_{0}(\omega) \|_{p}^{p}\right) d \tau .\right.\right.
\end{aligned}
$$

Replacing $\omega$ by $\theta_{-t-1} \omega$, and applying the Gagliardo-Nirenberg inequality and Young's inequality, 
we obtain, by Lemma 4.5 , for all $t \geq T_{0_{B}}(\omega)+1$,

$$
\begin{aligned}
\| \nabla v( & \left.+1, \theta_{-t-1} \omega, v_{0}\left(\theta_{-t-1} \omega\right)\right) \|^{2} \\
\leq & \int_{t}^{t+1}\left\|\nabla v\left(s, \theta_{-t-1} \omega, v_{0}\left(\theta_{-t-1} \omega\right)\right)\right\|^{2} d s+\int_{t}^{t+1} \eta_{3}\left(\theta_{\tau-t-1} \omega\right) d \tau \\
& +c \int_{t}^{t+1}\left(\| \nabla u\left(\tau, \theta_{-t-1} \omega, v_{0}\left(\theta_{-t-1} \omega\right)\left\|^{2}+\right\| u\left(\tau, \theta_{-t-1} \omega, v_{0}\left(\theta_{-t-1} \omega\right) \|_{p}^{p}\right) d \tau\right.\right. \\
\leq & \int_{t}^{t+1}\left\|(-\triangle)^{\alpha} v\left(s, \theta_{-t-1} \omega, v_{0}\left(\theta_{-t-1} \omega\right)\right)\right\|^{2} d s+\int_{t}^{t+1} \eta_{3}\left(\theta_{\tau-t-1} \omega\right) d \tau \\
& +c \int_{t}^{t+1}\left(\|(-\triangle)^{\alpha} u\left(\tau, \theta_{-t-1} \omega, v_{0}\left(\theta_{-t-1} \omega\right)\left\|^{2}+\right\| u\left(\tau, \theta_{-t-1} \omega, v_{0}\left(\theta_{-t-1} \omega\right) \|_{p}^{p}\right) d \tau\right.\right. \\
& +c \int_{t}^{t+1}\left(\| u\left(\tau, \theta_{-t-1} \omega, v_{0}\left(\theta_{-t-1} \omega\right)\left\|^{2}+\right\| v\left(\tau, \theta_{-t-1} \omega, v_{0}\left(\theta_{-t-1} \omega\right) \|^{2}\right) d \tau\right.\right. \\
\leq & c(r(\omega)+1)+\int_{-1}^{0} \eta_{3}\left(\theta_{\tau-t-1} \omega\right) d \tau \leq c(r(\omega)+1)+c \int_{-1}^{0}\left(e^{-\frac{\lambda}{2} \tau} r(\omega)+1\right) d \tau \\
\leq & c(r(\omega)+1) .
\end{aligned}
$$

By (4.61) and the definition of $z$, one has, for all $t \geq T_{0_{B}}(\omega)+1$,

$$
\begin{aligned}
\left\|\nabla u\left(t+1, \theta_{-t-1} \omega, u_{0}\left(\theta_{-t-1} \omega\right)\right)\right\|^{2} & \leq 2\left(\left\|\nabla v\left(t+1, \theta_{-t-1} \omega, v_{0}\left(\theta_{-t-1} \omega\right)\right)\right\|^{2}+\|\nabla z(\omega)\|^{2}\right) \\
& \leq c(r(\omega)+1) .
\end{aligned}
$$

Now we estimate far-field values of $v$ in the mean, for sufficiently large time.

Lemma 4.7. Suppose that (1.4)-(1.7) hold. Let $B=\{B(\omega)\} \in \mathcal{D}$ and $v_{0}(\omega) \in B(\omega)$. Then, for every $\varepsilon>0$ and $\omega \in \Omega$, there exist $T^{*}=T_{B}^{*}(\omega, \varepsilon)>0$ and $R^{*}=R^{*}(\omega, \varepsilon)>0$ such that for all $t \geq T_{B}^{*}(\omega)$, the solution $v\left(t, \omega, v_{0}(\omega)\right)$ of problem (3.5)-(3.6) satisfies

$$
\int_{|x| \geq R^{*}}\left|v\left(t, \theta_{-t} \omega, v_{0}\left(\theta_{-t} \omega\right)\right)\right|^{2} d x \leq \varepsilon
$$

Proof. Take a smooth $\chi$ such that $0 \leq \chi(s) \leq 1$ for all $s \geq 0$ and

$$
\chi(s)= \begin{cases}0, & \text { if } 0 \leq s \leq 1, \\ 1, & \text { if } s \geq 2 .\end{cases}
$$

Then there exists a positive constant $c$ such that $\left|\chi^{\prime}(s)\right| \leq c$ for all $s \geq 0$. Taking the inner product in $L^{2}$ of (3.5) with $\chi\left(\frac{|x|^{2}}{k^{2}}\right) v$, we obtain

$$
\begin{aligned}
\frac{1}{2} \frac{d}{d t} \int_{\mathbb{R}^{n}} \chi\left(\frac{|x|^{2}}{k^{2}}\right) & |v|^{2} d x+\int_{\mathbb{R}^{n}}(-\triangle)^{\alpha} v \chi\left(\frac{|x|^{2}}{k^{2}}\right) v d x+\lambda \int_{\mathbb{R}^{n}} \chi\left(\frac{|x|^{2}}{k^{2}}\right)|v|^{2} d x \\
& =\int_{\mathbb{R}^{n}} F(x, u) \chi\left(\frac{|x|^{2}}{k^{2}}\right) v d x+\int_{\mathbb{R}^{n}}(-\triangle)^{\alpha} z\left(\theta_{t} \omega\right) \chi\left(\frac{|x|^{2}}{k^{2}}\right) v d x
\end{aligned}
$$


Now, we estimate the terms in (4.64) as follows. For the second term on the left-hand side, integrating by parts and applying Hölder's, Young's, and the Gagliardo-Nirenberg inequality, we have

$$
\begin{aligned}
-\int_{\mathbb{R}^{n}} & (-\triangle)^{\alpha} v \chi\left(\frac{|x|^{2}}{k^{2}}\right) v d x \\
& \leq \int_{\mathbb{R}^{n}}\left|(-\triangle)^{\alpha-\frac{1}{2}} v\right|\left(\chi\left(\frac{|x|^{2}}{k^{2}}\right)|\nabla v|+\chi^{\prime}\left(\frac{x^{2}}{k^{2}}\right) \frac{2|x|}{k^{2}}|v|\right) d x \\
& \leq\left\|(-\triangle)^{\alpha-\frac{1}{2}} v\right\|\|\nabla v\|+\int_{k \leq|x| \leq \sqrt{2} k}\left|(-\triangle)^{\alpha-\frac{1}{2}} v\right|\left|\chi^{\prime}\left(\frac{|x|^{2}}{k^{2}}\right)\right| \frac{2|x|}{k^{2}}|v| d x \\
& \leq\left\|(-\triangle)^{\alpha-\frac{1}{2}} v\right\|\|\nabla v\|+\frac{2 \sqrt{2}}{k} \int_{k \leq|x| \leq \sqrt{2} k}\left|(-\triangle)^{\alpha-\frac{1}{2}} v\right|\left|\chi^{\prime}\left(\frac{|x|^{2}}{k^{2}}\right)\right||v| d x \\
& \leq\left\|(-\triangle)^{\alpha-\frac{1}{2}} v\right\|\|\nabla v\|+\frac{c}{k} \int_{k \leq|x| \leq \sqrt{2} k}\left|(-\triangle)^{\alpha-\frac{1}{2}} v\right||v| d x \\
& \leq c\left(\|v\|^{2}+\|\nabla v\|^{2}\right)+\frac{c}{k}\left(\|\nabla v\|^{2}+\|v\|^{2}\right) .
\end{aligned}
$$

For the first term on the right-hand side of (4.64), applying (1.4) and (1.5), one has

$$
\begin{aligned}
\int_{\mathbb{R}^{n}} F(x, u) \chi & \left(\frac{|x|^{2}}{k^{2}}\right) v d x=\int_{\mathbb{R}^{n}} F(x, u) \chi\left(\frac{|x|^{2}}{k^{2}}\right) u d x-\int_{\mathbb{R}^{n}} F(x, u) \chi\left(\frac{|x|^{2}}{k^{2}}\right) z\left(\theta_{t} \omega\right) d x \\
\leq & -\beta_{1} \int_{\mathbb{R}^{n}}|u|^{p} \chi\left(\frac{|x|^{2}}{k^{2}}\right) d x+\beta_{2} \int_{\mathbb{R}^{n}}|u|^{p-1} \chi\left(\frac{|x|^{2}}{k^{2}}\right)\left|z\left(\theta_{t} \omega\right)\right| d x \\
& +\int_{\mathbb{R}^{n}} \gamma_{1}(x) \chi\left(\frac{|x|^{2}}{k^{2}}\right) d x+\int_{\mathbb{R}^{n}} \gamma_{2}(x) \chi\left(\frac{|x|^{2}}{k^{2}}\right)\left|z\left(\theta_{t} \omega\right)\right| d x \\
\leq & -\beta_{1} \int_{\mathbb{R}^{n}}|u|^{p} \chi\left(\frac{|x|^{2}}{k^{2}}\right) d x+\frac{1}{2} \beta_{1} \int_{\mathbb{R}^{n}}|u|^{p} \chi\left(\frac{|x|^{2}}{k^{2}}\right) d x \\
& +\int_{\mathbb{R}^{n}} \gamma_{1}(x) \chi\left(\frac{|x|^{2}}{k^{2}}\right) d x+c \int_{\mathbb{R}^{n}}\left|z\left(\theta_{t} \omega\right)\right|^{p} \chi\left(\frac{|x|^{2}}{k^{2}}\right) d x \\
& +\frac{1}{2} \int_{\mathbb{R}^{n}}\left|z\left(\theta_{t} \omega\right)\right|^{2} \chi\left(\frac{|x|^{2}}{k^{2}}\right) d x+\frac{1}{2} \int_{\mathbb{R}^{n}} \gamma_{2}^{2}(x) \chi\left(\frac{|x|^{2}}{k^{2}}\right) d x \\
\leq & -\frac{1}{2} \beta_{1} \int_{\mathbb{R}^{n}}|u|^{p} \chi\left(\frac{|x|^{2}}{k^{2}}\right) d x+\int_{\mathbb{R}^{n}} \gamma_{1}(x) \chi\left(\frac{|x|^{2}}{k^{2}}\right) d x \\
& +\frac{1}{2} \int_{\mathbb{R}^{n}} \gamma_{2}^{2}(x) \chi\left(\frac{|x|^{2}}{k^{2}}\right) d x+c \int_{\mathbb{R}^{n}}\left(\left|z\left(\theta_{t} \omega\right)\right|^{2}+\left|z\left(\theta_{t} \omega\right)\right|^{p}\right) \chi\left(\frac{|x|^{2}}{k^{2}}\right) d x .
\end{aligned}
$$

For the second term on the right-hand side of (4.64), applying Young's inequality, one has

$$
\begin{aligned}
\int_{\mathbb{R}^{n}}(-\triangle)^{\alpha} & z\left(\theta_{t} \omega\right) \chi\left(\frac{|x|^{2}}{k^{2}}\right) v d x \\
& \leq \frac{1}{2} \lambda \int_{\mathbb{R}^{n}} \chi\left(\frac{|x|^{2}}{k^{2}}\right)|v|^{2} d x+\frac{1}{\lambda} \int_{\mathbb{R}^{n}}\left|(-\triangle)^{\alpha} z\left(\theta_{t} \omega\right)\right|^{2} \chi\left(\frac{|x|^{2}}{k^{2}}\right) d x
\end{aligned}
$$


By (4.65), (4.66) and (4.67), one has

$$
\begin{aligned}
\frac{d}{d t} \int_{\mathbb{R}^{n}} \chi & \left(\frac{|x|^{2}}{k^{2}}\right)|v|^{2} d x+\lambda \int_{\mathbb{R}^{n}} \chi\left(\frac{|x|^{2}}{k^{2}}\right)|v|^{2} d x+\beta_{1} \int_{\mathbb{R}^{n}}|u|^{p} \chi\left(\frac{|x|^{2}}{k^{2}}\right) d x \\
\leq & c\left(\|v\|^{2}+\|\nabla v\|^{2}\right)+\frac{c}{k}\left(\|\nabla v\|^{2}+\|v\|^{2}\right)+\int_{\mathbb{R}^{n}}\left(2\left|\gamma_{1}(x)\right|+\left|\gamma_{2}(x)\right|^{2}\right) \chi\left(\frac{|x|^{2}}{k^{2}}\right) d x \\
& +c \int_{\mathbb{R}^{n}}\left(\left|(-\triangle)^{\alpha} z\left(\theta_{t} \omega\right)\right|^{2}+\left|z\left(\theta_{t} \omega\right)\right|^{2}+\left|z\left(\theta_{t} \omega\right)\right|^{p}\right) \chi\left(\frac{|x|^{2}}{k^{2}}\right) d x .
\end{aligned}
$$

By Lemmas 4.1 and 4.6, there exists $T_{1}=T_{1}(B, \omega)>0$ such that for all $t \geq T_{1}$,

$$
\left\|v\left(t, \theta_{-t} \omega, v_{0}\left(\theta_{-t} \omega\right)\right)\right\|_{H^{1}\left(\mathbb{R}^{n}\right)}^{2} \leq c(r(\omega)+1) .
$$

Integrating (4.68) over $\left(T_{1}, t\right)$, we infer that, for all $t \geq T_{1}$,

$$
\begin{aligned}
\int_{\mathbb{R}^{n}} \chi\left(\frac{|x|^{2}}{k^{2}}\right) \mid v & \left.\left(t, \omega, v_{0}(\omega)\right)\right|^{2} d x \leq e^{\lambda\left(T_{1}-t\right)} \int_{\mathbb{R}^{n}} \chi\left(\frac{|x|^{2}}{k^{2}}\right)\left|v\left(T_{1}, \omega, v_{0}(\omega)\right)\right|^{2} d x \\
& +\int_{T_{1}}^{t} e^{\lambda(s-t)} \int_{\mathbb{R}^{n}}\left(2\left|\gamma_{1}(x)\right|+\left|\gamma_{2}(x)\right|^{2}\right) \chi\left(\frac{|x|^{2}}{k^{2}}\right) d x d s \\
& +\int_{T_{1}}^{t} e^{\lambda(s-t)} \int_{\mathbb{R}^{n}}\left(\left|(-\triangle)^{\alpha} z\left(\theta_{s} \omega\right)\right|^{2}+\left|z\left(\theta_{s} \omega\right)\right|^{2}+\left|z\left(\theta_{s} \omega\right)\right|^{p}\right) \chi\left(\frac{|x|^{2}}{k^{2}}\right) d x d s \\
& +c \int_{T_{1}}^{t} e^{\lambda(s-t)}\left(\left\|v\left(s, \omega, v_{0}(\omega)\right)\right\|^{2}+\left\|\nabla v\left(s, \omega, v_{0}(\omega)\right)\right\|^{2}\right) d s \\
& +\frac{c}{k} \int_{T_{1}}^{t} e^{\lambda(s-t)}\left(\left\|\nabla v\left(s, \omega, v_{0}(\omega)\right)\right\|^{2}+\left\|v\left(s, \omega, v_{0}(\omega)\right)\right\|^{2}\right) d s
\end{aligned}
$$

Replacing $\omega$ by $\theta_{-t} \omega$ in (4.70), one has, for all $t \geq T_{1}$,

$$
\begin{aligned}
\int_{\mathbb{R}^{n}} \chi( & \left.\frac{|x|^{2}}{k^{2}}\right)\left|v\left(t, \theta_{-t} \omega, v_{0}\left(\theta_{-t} \omega\right)\right)\right|^{2} d x \leq e^{\lambda\left(T_{1}-t\right)} \int_{\mathbb{R}^{n}} \chi\left(\frac{|x|^{2}}{k^{2}}\right)\left|v\left(T_{1}, \theta_{-t} \omega, v_{0}\left(\theta_{-t} \omega\right)\right)\right|^{2} d x \\
& +\int_{T_{1}}^{t} e^{\lambda(s-t)} \int_{\mathbb{R}^{n}}\left(2\left|\gamma_{1}(x)\right|+\left|\gamma_{2}(x)\right|^{2}\right) \chi\left(\frac{|x|^{2}}{k^{2}}\right) d x d s \\
& +\int_{T_{1}}^{t} e^{\lambda(s-t)} \int_{\mathbb{R}^{n}}\left(\left|(-\triangle)^{\alpha} z\left(\theta_{s-t} \omega\right)\right|^{2}+\left|z\left(\theta_{s-t} \omega\right)\right|^{2}+\left|z\left(\theta_{s-t} \omega\right)\right|^{p}\right) \chi\left(\frac{|x|^{2}}{k^{2}}\right) d x d s \\
& +c \int_{T_{1}}^{t} e^{\lambda(s-t)}\left(\left\|v\left(s, \theta_{-t} \omega, v_{0}\left(\theta_{-t} \omega\right)\right)\right\|^{2}+\left\|\nabla v\left(s, \theta_{-t} \omega, v_{0}\left(\theta_{-t} \omega\right)\right)\right\|^{2}\right) d s \\
& +\frac{c}{k} \int_{T_{1}}^{t} e^{\lambda(s-t)}\left\|\nabla v\left(s, \theta_{-t} \omega, v_{0}\left(\theta_{-t} \omega\right)\right)\right\|^{2} d s \\
& +\frac{c}{k} \int_{T_{1}}^{t} e^{\lambda(s-t)}\left\|v\left(s, \theta_{-t} \omega, v_{0}\left(\theta_{-t} \omega\right)\right)\right\|^{2} d s .
\end{aligned}
$$

In what follows, we estimate each term on the right-hand side of (4.71). For the first term, replacing 
$t$ by $T_{1}$ and replacing $\omega$ by $\theta_{-t} \omega$ in (4.15), we have

$$
\begin{aligned}
e^{\lambda\left(T_{1}-t\right)} & \int_{\mathbb{R}^{n}} \chi\left(\frac{|x|^{2}}{k^{2}}\right)\left|v\left(T_{1}, \theta_{-t} \omega, v_{0}\left(\theta_{-t} \omega\right)\right)\right|^{2} d x \\
& \leq e^{\lambda\left(T_{1}-t\right)}\left(e^{-\lambda T_{1}}\left\|v_{0}\left(\theta_{-t} \omega\right)\right\|^{2} d x+\int_{0}^{T_{1}} e^{\lambda\left(s-T_{1}\right)} \eta_{1}\left(\theta_{s-t} \omega\right) d s+\frac{c_{7}}{\lambda}\right) \\
& \leq e^{-\lambda t}\left\|v_{0}\left(\theta_{-t} \omega\right)\right\|^{2} d x+c e^{\lambda\left(T_{1}-t\right)}+\int_{-t}^{T_{1}-t} e^{\lambda \tau} \eta_{1}\left(\theta_{\tau} \omega\right) d \tau \\
& \leq e^{-\lambda t}\left\|v_{0}\left(\theta_{-t} \omega\right)\right\|^{2} d x+c e^{\lambda\left(T_{1}-t\right)}+c_{6} r(\omega) \int_{-t}^{T_{1}-t} e^{\frac{1}{2} \lambda \tau} d \tau \\
& \leq e^{-\lambda t}\left\|v_{0}\left(\theta_{-t} \omega\right)\right\|^{2} d x+c e^{\lambda\left(T_{1}-t\right)}+\frac{2}{\lambda} c_{6} r(\omega) e^{\frac{1}{2} \lambda\left(T_{1}-t\right)}
\end{aligned}
$$

Therefore, given $\varepsilon>0$, there exists $T_{2}=T_{2}(B, \omega, \varepsilon)>T_{1}$ such that for all $t \geq T_{2}$,

$$
e^{\lambda\left(T_{1}-t\right)} \int_{\mathbb{R}^{n}} \chi\left(\frac{|x|^{2}}{k^{2}}\right)\left|v\left(T_{1}, \theta_{-t} \omega, v_{0}\left(\theta_{-t} \omega\right)\right)\right|^{2} d x \leq \frac{\varepsilon}{6} .
$$

For the second term, note that $\gamma_{1}(x) \in L^{1}\left(\mathbb{R}^{n}\right)$ and $\gamma_{2}(x) \in L^{2}\left(\mathbb{R}^{n}\right)$, so there exists $R_{1}=R_{1}(\varepsilon)$ such that for all $k \geq R_{1}$, we have

$$
\int_{|x| \geq k}\left(2\left|\gamma_{1}(x)\right|+\left|\gamma_{2}(x)\right|^{2}\right) \chi\left(\frac{|x|^{2}}{k^{2}}\right) d x \leq \frac{\lambda \varepsilon}{6}
$$

and so one has

$$
\begin{aligned}
\int_{T_{1}}^{t} e^{\lambda(s-t)} \int_{\mathbb{R}^{n}}\left(2\left|\gamma_{1}(x)\right|+\right. & \left.\left|\gamma_{2}(x)\right|^{2}\right) \chi\left(\frac{|x|^{2}}{k^{2}}\right) d x d s \\
& \leq \int_{T_{1}}^{t} e^{\lambda(s-t)} \int_{|x| \geq k}\left(2\left|\gamma_{1}(x)\right|+\left|\gamma_{2}(x)\right|^{2}\right) d x d s \\
& \leq \frac{\varepsilon}{6} .
\end{aligned}
$$

Note that

$$
z\left(\theta_{t} \omega\right)=\sum_{j=1}^{m} \phi_{j} z_{j}\left(\theta_{t} \omega_{j}\right) \text { and } \phi_{j} \in H^{2}\left(\mathbb{R}^{n}\right) \cap W^{2, p}\left(\mathbb{R}^{n}\right) .
$$

Hence, there exists $R_{2}=R_{2}(\omega, \varepsilon)$ such that for all $k \geq R_{2}$ and $j=1,2, \ldots, m$,

$$
\int_{|x| \geq k}\left(\left|\phi_{j}\right|^{2}+\left|\phi_{j}\right|^{p}+\left|(-\triangle)^{\alpha} \phi_{j}\right|^{2}\right) d x \leq \frac{1}{6} \min \left\{\frac{\lambda \varepsilon}{4 m^{p} r(\omega)}, \frac{\varepsilon}{2 m r(\omega)}\right\},
$$


where $r(\omega)$ is the tempered function in (3.2). By (4.76) and (3.2)-(3.3), for the third term, we have

$$
\begin{aligned}
& \int_{T_{1}}^{t} e^{\lambda(s-t)} \int_{\mathbb{R}^{n}}\left(\left|(-\triangle)^{\alpha} z\left(\theta_{s-t} \omega\right)\right|^{2}+\left|z\left(\theta_{s-t} \omega\right)\right|^{2}+\left|z\left(\theta_{s-t} \omega\right)\right|^{p}\right) \chi\left(\frac{|x|^{2}}{k^{2}}\right) d x d s \\
& \leq \int_{T_{1}}^{t} e^{\lambda(s-t)} \int_{|x| \geq k}\left(\left|(-\triangle)^{\alpha} z\left(\theta_{s-t} \omega\right)\right|^{2}+\left|z\left(\theta_{s-t} \omega\right)\right|^{2}+\left|z\left(\theta_{s-t} \omega\right)\right|^{p}\right) d x d s \\
& \leq \int_{T_{1}}^{t} e^{\lambda(s-t)} \sum_{j=1}^{m} \int_{|x| \geq k}\left(m\left(\left|(-\triangle)^{\alpha} \phi_{j}\right|^{2}+\left|\phi_{j}\right|^{2}\right)\left|z_{j}\left(\theta_{s-t} \omega_{j}\right)\right|^{2}+m^{p}\left|\phi_{j}\right|^{p}\left|z_{j}\left(\theta_{s-t} \omega_{j}\right)\right|^{p}\right) d x d s \\
& \leq \frac{\lambda \varepsilon}{12 r(\omega)} \int_{T_{1}}^{t} e^{\lambda(s-t)} \sum_{j=1}^{m}\left(\left|z_{j}\left(\theta_{s-t} \omega_{j}\right)\right|^{2}+\left|z_{j}\left(\theta_{s-t} \omega_{j}\right)\right|^{p}\right) d s \leq \frac{\lambda \varepsilon}{12 r(\omega)} \int_{T_{1}}^{t} e^{\lambda(s-t)} r\left(\theta_{s-t} \omega\right) d s \\
& \leq \frac{\lambda \varepsilon}{12 r(\omega)} \int_{T_{1}-t}^{0} e^{\lambda s} r\left(\theta_{s} \omega\right) d s \leq \frac{\lambda \varepsilon}{12 r(\omega)} \int_{T_{1}-t}^{0} e^{\frac{1}{2} \lambda s} r(\omega) d s \\
& \leq \frac{\varepsilon}{6} .
\end{aligned}
$$

For the fourth term, by Lemmas 4.1 and 4.6, there exists $T_{3}=T_{3}(B, \omega, \varepsilon)>T_{1}$ such that

$$
c \int_{T_{1}}^{t} e^{\lambda(s-t)}\left(\left\|v\left(s, \theta_{-t} \omega, v_{0}\left(\theta_{-t} \omega\right)\right)\right\|^{2}+\left\|\nabla v\left(s, \theta_{-t} \omega, v_{0}\left(\theta_{-t} \omega\right)\right)\right\|^{2}\right) d s \leq \frac{\varepsilon}{6}, \text { for all } t \geq T_{3} .
$$

By Lemma 4.6, there exists $T_{4}=T_{4}(B, \omega)>T_{1}$ such that the fifth term on the right-side hand of (4.71) satisfies

$$
\frac{c}{k} \int_{T_{1}}^{t} e^{\lambda(s-t)}\left\|\nabla v\left(s, \theta_{-t} \omega, v_{0}\left(\theta_{-t} \omega\right)\right)\right\|^{2} d s \leq \frac{c}{k}(r(\omega)+1) .
$$

Then, there is $R_{3}=R_{3}(\omega, \varepsilon)$ such that for all $t \geq T_{4}$ and $k \geq R_{3}$,

$$
\frac{c}{k} \int_{T_{1}}^{t} e^{\lambda(s-t)}\left\|\nabla v\left(s, \theta_{-t} \omega, v_{0}\left(\theta_{-t} \omega\right)\right)\right\|^{2} d s \leq \frac{\varepsilon}{6} .
$$

For the last term, first replacing $t$ by $\tau$ and then replacing $\omega$ by $\theta_{-t} \omega$ in (4.15), we deduce that

$$
\begin{aligned}
\frac{c}{k} & \int_{T_{1}}^{t} e^{\lambda(\tau-t)}\left\|v\left(\tau, \theta_{-t} \omega, v_{0}\left(\theta_{-t} \omega\right)\right)\right\|^{2} d s \\
& \leq \frac{c}{k} \int_{T_{1}}^{t} e^{-\lambda t}\left\|v_{0}\left(\theta_{-t} \omega\right)\right\|^{2} d \tau+\frac{c}{k} \int_{T_{1}}^{t} e^{\lambda(\tau-t)} \int_{0}^{\tau} e^{\lambda(s-\tau)} \eta_{1}\left(\theta_{s-t} \omega\right) d s d \tau+\frac{c}{k} \int_{T_{1}}^{t} e^{\lambda(\tau-t)} d \tau \\
& \leq \frac{c}{k} e^{-\lambda t}\left(t-T_{1}\right)\left\|v_{0}\left(\theta_{-t} \omega\right)\right\|^{2}+\frac{c}{k} \int_{T_{1}}^{t} e^{\lambda(\tau-t)} \int_{0}^{\tau} e^{\lambda(s-\tau)} \eta_{1}\left(\theta_{s-t} \omega\right) d s d \tau+\frac{c}{k} \\
& \leq \frac{c}{k} e^{-\lambda t}\left(t-T_{1}\right)\left\|v_{0}\left(\theta_{-t} \omega\right)\right\|^{2}+\frac{c}{k} \int_{T_{1}}^{t} e^{\lambda(\tau-t)} \int_{-t}^{\tau-t} e^{\lambda s} \eta_{1}\left(\theta_{s} \omega\right) d s d \tau+\frac{c}{k} \\
& \leq \frac{c}{k} e^{-\lambda t}\left(t-T_{1}\right)\left\|v_{0}\left(\theta_{-t} \omega\right)\right\|^{2}+\frac{c}{k} c_{6} r(\omega) \int_{T_{1}}^{t} e^{\lambda(\tau-t)} \int_{-t}^{\tau-t} e^{\frac{1}{2} \lambda s} d s d \tau+\frac{c}{k} \\
& \leq \frac{c}{k} e^{-\lambda t}\left(t-T_{1}\right)\left\|v_{0}\left(\theta_{-t} \omega\right)\right\|^{2}+\frac{4 c}{\lambda^{2} k} c_{6} r(\omega)+\frac{c}{k}
\end{aligned}
$$


This implies that there is $T_{5}=T_{5}(B, \omega, \varepsilon)>T_{1}$ and $R_{4}=R_{4}(\omega, \varepsilon)$ such that for all $t \geq T_{5}$ and $k \geq R_{4}$,

$$
\frac{c}{k} \int_{T_{1}}^{t} e^{\lambda(s-t)}\left\|v\left(s, \theta_{-t} \omega, v_{0}\left(\theta_{-t} \omega\right)\right)\right\|^{2} d s \leq \frac{\varepsilon}{6} .
$$

Let $T^{*}=T^{*}(B, \omega, \varepsilon)=\max \left\{T_{1}, T_{2}, T_{3}, T_{4}, T_{5}\right\}$. By (4.73), (4.75), (4.77), (4.78), (4.79) and (4.80), for all $t \geq T^{*}$ and $k \geq R^{*}=\max \left\{R_{1}, R_{2}, R_{3}, R_{4}\right\}$, one has

$$
\int_{\mathbb{R}^{n}} \chi\left(\frac{x^{2}}{k^{2}}\right)\left|v\left(t, \theta_{-t} \omega, v_{0}\left(\theta_{-t} \omega\right)\right)\right|^{2} d x \leq \varepsilon
$$

This implies that for all $t \geq T^{*}$ and $k \geq R^{*}$, one has

$$
\int_{|x| \geq k}\left|v\left(t, \theta_{-t} \omega, v_{0}\left(\theta_{-t} \omega\right)\right)\right|^{2} d x \leq \int_{\mathbb{R}^{n}} \chi\left(\frac{x^{2}}{k^{2}}\right)\left|v\left(t, \theta_{-t} \omega, v_{0}\left(\theta_{-t} \omega\right)\right)\right|^{2} d x \leq \varepsilon,
$$

completing the proof.

This now gives a similar result for $u$.

Lemma 4.8. Suppose that (1.4)-(1.7) hold. Let $B=\{B(\omega)\} \in \mathcal{D}$ and $u_{0}(\omega) \in B(\omega)$. Then for every $\varepsilon>0$ and $\omega \in \Omega$, there exist $T^{*}=T_{B}^{*}(\omega, \varepsilon)>0$ and $R^{*}=R^{*}(\omega, \varepsilon)>0$ such that, for all $t \geq T_{B}^{*}(\omega)$, one has

$$
\int_{|x| \geq R^{*}}\left|u\left(t, \theta_{-t} \omega, u_{0}\left(\theta_{-t} \omega\right)\right)\right|^{2} d x \leq \varepsilon .
$$

Proof. Let $T^{*}$ and $R^{*}$ be the constants in Lemma 4.7. By (4.76) and (3.2), for all $t \geq T^{*}$,

$$
\begin{aligned}
\int_{|x| \geq R^{*}}|z(\omega)|^{2} d x & =\int_{|x| \geq R^{*}}\left|\sum_{j=1}^{m} \phi_{j} z_{j}\left(\omega_{j}\right)\right|^{2} d x \\
& \leq m \int_{|x| \geq R^{*}} \sum_{j=1}^{m}\left|\phi_{j}\right|^{2}\left|z_{j}\left(\omega_{j}\right)\right|^{2} d x \leq \frac{\varepsilon}{12 r(\omega)} \sum_{j=1}^{m}\left|z_{j}\left(\omega_{j}\right)\right|^{2} \\
& \leq \frac{\varepsilon}{12}
\end{aligned}
$$

Applying Lemma 4.7, we obtain, for all $t \geq T^{*}$,

$$
\begin{aligned}
\int_{|x| \geq R^{*}}\left|u\left(t, \theta_{-t} \omega, u_{0}\left(\theta_{-t} \omega\right)\right)\right|^{2} d x & =\int_{|x| \geq R^{*}}\left|v\left(t, \theta_{-t} \omega, v_{0}\left(\theta_{-t} \omega\right)\right)+z(\omega)\right|^{2} d x \\
& \leq 2 \int_{|x| \geq R^{*}}\left|v\left(t, \theta_{-t} \omega, v_{0}\left(\theta_{-t} \omega\right)\right)\right|^{2} d x+2 \int_{|x| \geq R^{*}}|z(\omega)|^{2} \\
& \leq 3 \varepsilon
\end{aligned}
$$

This completes the proof. 


\section{Random attractor}

In this section, we prove the existence of a random attractor for the random dynamical system generated by (3.5) on $\mathbb{R}^{n}$. From Lemma 4.1, we have that $\varphi$ has a closed random absorbing set in $\mathcal{D}$. The $\mathcal{D}$-pullback asymptotic compactness of $\varphi$ is demonstrated below using the uniform estimates obtained in the previous sections.

Lemma 5.1. Suppose that (1.4)-(1.7) hold. Then the random dynamical system $\varphi$ is $\mathcal{D}$-pullback asymptotically compact in $L^{2}\left(\mathbb{R}^{n}\right)$; that is, for $\omega \in \Omega$, the sequence $\varphi\left(t_{n}, \theta_{-t_{n}} \omega, v_{0, n}\left(\theta_{-t_{n}} \omega\right)\right)$ has a convergent subsequence in $L^{2}\left(\mathbb{R}^{n}\right)$ provided $t_{n} \rightarrow \infty, B=\{B(\omega)\} \in \mathcal{D}$ and $v_{0, n}\left(\theta_{-t_{n}} \omega\right) \in B\left(\theta_{-t_{n}} \omega\right)$.

Proof. Let $t_{n} \rightarrow \infty, B=\{B(\omega)\} \in \mathcal{D}$ and $v_{0, n}\left(\theta_{-t_{n}} \omega\right) \in B\left(\theta_{-t_{n}} \omega\right)$. Applying Lemma 4.1, for all $\omega \in \Omega$, we have

$$
\left\{\varphi\left(t_{n}, \theta_{-t_{n}} \omega, v_{0, n}\left(\theta_{-t_{n}} \omega\right)\right)\right\}_{n=1}^{\infty} \quad \text { is bounded in } L^{2}\left(\mathbb{R}^{n}\right)
$$

Therefore, there exists $\eta \in L^{2}\left(\mathbb{R}^{n}\right)$ and a subsequence, still denoted by $\left\{\varphi\left(t_{n}, \theta_{-t_{n}} \omega, v_{0, n}\left(\theta_{-t_{n}} \omega\right)\right)\right\}$, such that

$$
\varphi\left(t_{n}, \theta_{-t_{n}} \omega, v_{0, n}\left(\theta_{-t_{n}} \omega\right)\right) \rightarrow \eta \quad \text { weakly in } L^{2}\left(\mathbb{R}^{n}\right), \text { for some } \eta=\eta(\omega) \in L^{2}\left(\mathbb{R}^{n}\right)
$$

By Lemma 4.7, there is $T^{*}=T_{B}^{*}(\omega)$ and $R^{*}=R^{*}(\omega, \varepsilon)$ such that for all $t \geq T^{*}$,

$$
\left\|\varphi\left(t, \theta_{-t} \omega, v_{0}\left(\theta_{-t} \omega\right)\right)\right\|_{L^{2}\left(|x| \geq R^{*}\right)}^{2} \leq \varepsilon
$$

Since $t_{n} \rightarrow \infty$, there exists $N_{1}=N_{1}(B, \omega, \varepsilon)$ such that $t_{n} \geq T_{B}^{*}$ for all $n \geq N_{1}$. By (5.1), for all $n \geq N_{1}$, one has

$$
\left\|\varphi\left(t_{n}, \theta_{-t_{n}} \omega, v_{0, n}\left(\theta_{-t_{n}} \omega\right)\right)\right\|_{L^{2}\left(|x| \geq R^{*}\right)}^{2} \leq \varepsilon
$$

Applying Lemmas 4.1 and 4.6, there exists $T_{1_{B}}=T_{0_{B}}(\omega)+1$ such that for all $t \geq T_{1_{B}}$,

$$
\left\|\varphi\left(t, \theta_{-t} \omega, v_{0}\left(\theta_{-t} \omega\right)\right)\right\|_{H^{1}\left(\mathbb{R}^{n}\right)}^{2} \leq c(r(\omega)+1) .
$$

Let $N_{2}=N_{2}(B, \omega)$ be large enough such that $t_{n} \geq T_{1_{B}}$ and $n \geq N_{2}$. It follows from (5.2) that, for all $n \geq N_{2}$,

$$
\left\|\varphi\left(t_{n}, \theta_{-t_{n}} \omega, v_{0, n}\left(\theta_{-t_{n}} \omega\right)\right)\right\|_{H^{1}\left(\mathbb{R}^{n}\right)}^{2} \leq c(r(\omega)+1),
$$

and so a further subsequence converges weakly in $H^{1}\left(\mathbb{R}^{n}\right)$, again to $\eta$. Thus, $\eta \in H^{1}\left(\mathbb{R}^{n}\right)$. Let $\bar{B}_{R^{*}}=\left\{x \in \mathbb{R}^{n}:|x| \leq R^{*}\right\}$. By the compactness of the embedding $H^{1}\left(\bar{B}_{R^{*}}\right) \hookrightarrow L^{2}\left(\bar{B}_{R^{*}}\right)$, together 
with (5.3), we obtain, up to a subsequence depending on $R^{*}$,

$$
\varphi\left(t_{n}, \theta_{-t_{n}} \omega, v_{0, n}\left(\theta_{-t_{n}} \omega\right)\right) \rightarrow \eta \quad \text { strongly in } L^{2}\left(\bar{B}_{R^{*}}\right)
$$

which implies that there exists $N_{3}=N_{3}(B, \omega, \varepsilon)$ such that for all $n \geq N_{3}$,

$$
\left\|\varphi\left(t_{n}, \theta_{-t_{n}} \omega, v_{0, n}\left(\theta_{-t_{n}} \omega\right)\right)-\eta\right\|_{L^{2}\left(\bar{B}_{R^{*}}\right)}^{2} \leq \varepsilon
$$

Since $\eta \in L^{2}\left(\mathbb{R}^{n}\right)$, there exists $R^{* *}=R^{* *}(\varepsilon)>0$ such that

$$
\int_{|x| \geq R^{* *}}|\eta(x)|^{2} d x \leq \varepsilon .
$$

Let $R^{\star}=\max \left\{R^{*}, R^{* *}\right\}$ and $N^{\star}=\max \left\{N_{1}, N_{3}\right\}$. Then, from (5.1), (5.4) and (5.5), we obtain that for all $n \geq N^{\star}$,

$$
\begin{aligned}
& \left\|\varphi\left(t_{n}, \theta_{-t_{n}} \omega, v_{0, n}\left(\theta_{-t_{n}} \omega\right)\right)-\eta\right\|_{L^{2}\left(\mathbb{R}^{n}\right)}^{2} \\
& \quad \leq\left\|\varphi\left(t_{n}, \theta_{-t_{n}} \omega, v_{0, n}\left(\theta_{-t_{n}} \omega\right)\right)-\eta\right\|_{L^{2}\left(B_{R^{\star}}\right)}^{2}+\left\|\varphi\left(t_{n}, \theta_{-t_{n}} \omega, v_{0, n}\left(\theta_{-t_{n}} \omega\right)\right)-\eta\right\|_{L^{2}\left(B_{R^{\star}}^{c}\right)}^{2} \\
& \quad \leq 5 \varepsilon
\end{aligned}
$$

which implies $\varphi\left(t_{n}, \theta_{-t_{n}} \omega, v_{0, n}\left(\theta_{-t_{n}} \omega\right)\right) \rightarrow \eta$ strongly in $L^{2}\left(\mathbb{R}^{n}\right)$. This completes the proof.

Following from Proposition 2.1, we obtain

Theorem 5.1. Assume that (1.4)-(1.7) hold. The random dynamical system $\varphi$ associated with the fractional power dissipative equations with additive noise (1.2) has a unique $\mathcal{D}$-random attractor in $L^{2}\left(\mathbb{R}^{n}\right)$.

Remark 5.2. Note that the attractor lies in $H^{1}\left(\mathbb{R}^{n}\right)$ and attracts weakly in that norm. We have been unable to show strong attraction in that norm.

\section{Acknowledgements}

The authors were supported in part by the NSF DMS-0908348 and DMS-1413060, the NSF of China (No.11371183 and No. 11271050), NSF of Shandong Province (No. ZR2013AM004), and start-up funds for new faculties in New Mexico Institute of Mining and Technology. 


\section{References}

[1] L. Arnold, Random dynamical systems. Springer-Verlag, New York, 1998.

[2] A.V. Babin and M. I. Vishik, Attractors of evolution equations. North-Holland, Amsterdam, 1992.

[3] Z. Brzezniak and Y. Li, Asymptotic compactness and absorbing sets for 2D stochastic Navier-Stokes equations on some unbounded domains. Trans. American Math. Soc. 358 (2006), 5587-5629.

[4] P. W. Bates, H. Lisei and K. Lu, Attractors for stochastic lattice dynamical system. Stoch. Dyn. 6 (2006), 1-21.

[5] P. W. Bates, K. Lu and B. Wang, Random attractors for stochastic reaction-diffusion equations on unbounded domains. J. Differential Equations 246 (2009), 845-869.

[6] L. Caffarelli, S. Salsa and L. Silvestre, Regularity estimates for the solution and the free boundary of the obstacle problem for the fractional Laplacian. Invent. Math. 171 (2008), 425461.

[7] H. Crauel, A. Debussche and F. Flandoli, Random attractors. J. Dynam. Differential Equations 9 (1997), 307-341.

[8] H. Crauel and F. Flandoli, Attractors for random dynamical systems. Probab. Theory Related Fields 100 (1994), 365-393.

[9] T. Caraballo, J. A. Langa and J. C. Robinson, A stochastic pitchfork bifurcation in a reactiondiffusion equation. Proc. R. Soc. Lond. A 457 (2001), 2041-2061.

[10] A. Debussche, Hausdorff dimension of a random invariant set. J. Math. Pures Appl. 77 (1998), 967-988.

[11] J. Dong and M. Xu, Space-time fractional Schrödinger equation with time-independent potentials. J. Math. Anal. Appl. 344 (2008), 1005-1017.

[12] Z. E. A. Fellah, C. Depollier and M. Fellah, Propagation of ultrasonic pulses in porous elastic solids: a time domain analysis with fractional derivatives, 5th International Conference on 
Mathematical and Numerical Aspects of Wave Propagation. Santiago de Compostela, Spain, SIAM, Philadelphia, 2000.

[13] F. Flandoli and B. Schmalfuss, Random attractors for the 3D stochastic Navier-Stokes equation with multiplicative white noise. Stoch. Stoch. Rep. 59 (1996), 21-45.

[14] C.W. Gardiner, Handbooks of stochastic methods for physics, Chemistry and Natural Sciences. Springer-Verlag, Berlin, 1983.

[15] B. Guo and Z. Huo, Global well-posedness for the fractional nonlinear Schrödinger equation. Comm. Partial Differential Equations 36 (2011), 247-255.

[16] B. Guo, Y. Han and J. Xin, Existence of the global smooth solution to the period boundary value problem of fractional nonlinear Schrödinger equation. Appl. Math. Comput. 204 (2008), 468-477.

[17] S.G. Samko, A.A. Kilbas and O.I. Marichev, Fractional integrals and derivatives: Theory and applications. Gordon and Breach Science, New York, 1987.

[18] J.K. Hale, Asymptotic behavior of dissipative systems. Math. Surveys Monogr. 25 AMS, Providence, 1988.

[19] H. Lu, P. W. Bates, S. Lü and M. Zhang, Dynamics of 3D fractional complex GinzburgLandau equation. To appear in J. Differential Equations.

[20] E.W. Montroll and M.F. Shlesinger, On the wonderful world of random walks, in: J. Leibowitz and E.W. Montroll (Eds.), Nonequilibrium Phenomena II: from Stochastics to Hydrodynamics. North-Holland, Amsterdam, 1984, 1-121.

[21] C. Miao, B. Yuan and B. Zhang, Well-posedness of the Cauchy problem for the fractional power dissipative equations. Nonlinear Anal. 68 (2008), 461-484.

[22] R.R. Nigmatullin, The realization of the generalized transfer equation in a medium with fractal geometry. Phys. Stat. Sol. B 133 (1986), 425-430.

[23] L. Nirenberg, On elliptic partial differential equations. Ann. Scuola Norm. Sup. Pisa 13 (1959), 115-162. 
[24] X. Pu and B. Guo, Global weak solutions of the fractional Landau-Lifshitz-Maxwell equation. J. Math. Anal. Appl. 372 (2010), 86-98.

[25] J.C. Robinson, Infinite-dimensional dynamical systems. Cambridge Univ. Press, Cambridge, UK, 2001.

[26] S., Salsa, Optimal regularity in lower dimensional obstacle problems. Subelliptic PDE's and applications to geometry and finance. Lect. Notes Semin. Interdiscip. Mat., 6, Semin. Interdiscip. Mat. (S.I.M.), Potenza, 2007, 217-226.

[27] G. Sell and Y. You, Dynamics of evolutional equations. Springer-Verlag, New York, 2002.

[28] M. F. Shlesinger, G. M. Zaslavsky and J. Klafter, Strange kinetics. Nature 363 (1993), 31-37.

[29] A.I. Saichev and G.M. Zaslavsky, Fractional kinetic equations: solutions and applications. Chaos 7 (1997), 753-764.

[30] Y. Sire and E. Valdinoci, Fractional Laplacian phase transitions and boundary reactions: a geometric inequality and a symmetry result. J. Funct. Anal. 256 (2009), 1842-1864.

[31] R. Temam, Infinite dimension dynamical systems in mechanics and physics. SpringerVerlag, New York, 1995.

[32] V. E. Tarasov and G. M. Zaslavsky, Fractional Ginzburg-Landau equation for fractal media. Phys. A 354 (2005), 249-261.

[33] B. Wang, Asymptotic behavior of stochastic wave equations with critical exponents on $\mathbb{R}^{3}$. Trans. American Math. Soc. 363 (2011), 3639-3663.

[34] B. Wang and X. Gao, Random attractors for stochastic wave equations on unbounded domains. Discrete Contin. Dyn. Syst. Supplement (2009), 800-809.

[35] G. Wu and J. Yuan, Well-posedness of the Cauchy problem for the fractional power dissipative equation in critical Besov spaces. J. Math. Anal. Appl. 340 (2008), 1326-1335.

[36] G.M. Zaslavsky, Hamiltonian chaos and fractional dynamics. Oxford University Press, 2005.

[37] G.M. Zaslavsky, Chaos, fractional kinetics, and anomalous transport. Phys. Rep. 371 (2002), $461-580$. 
[38] G.M. Zaslavsky and M. Edelman, Weak mixing and anomalous kinetics along filamented surfaces. Chaos 11 (2001), 295-305. 